\title{
Frequency-dependent attenuation and dispersion caused by squirt flow: Three-dimensional numerical study
}

\author{
Yury Alkhimenkov ${ }^{1}$, Eva Caspari ${ }^{2}$, Boris Gurevich ${ }^{3}$, Nicolás D. Barbosa ${ }^{4}$, Stanislav \\ Glubokovskikh $^{5}$, Jürg Hunziker ${ }^{1}$, and Beatriz Quintal ${ }^{1}$
}

\begin{abstract}
Seismic waves may exhibit significant dispersion and attenuation in reservoir rocks due to pore-scale fluid flow. Fluid flow at the microscopic scale is referred to as squirt flow and occurs in very compliant pores, such as grain contacts or microcracks, that are connected to other stiffer pores. We have performed 3D numerical simulations of squirt flow using a finite-element approach. Our 3D numerical models consist of a pore space embedded into a solid grain material. The pore space is represented by a flat cylinder (a compliant crack) whose edge is connected with a torus (a stiff pore). Grains are described as a linear isotropic elastic material, whereas the fluid phase is described by the quasistatic linearized compressible Navier-Stokes momentum equation. We obtain the frequency-dependent effective
\end{abstract}

stiffness of a porous medium and calculate dispersion and attenuation due to fluid flow from a compliant crack to a stiff pore. We compare our numerical results against a published analytical solution for squirt flow and analyze the effects of its assumptions. Previous interpretation of the squirt flow phenomenon based mainly on analytical solutions is verified, and some new physical effects are identified. The numerical and analytical solutions agree only for the simplest model in which the edge of the crack is subjected to zero fluid pressure boundary condition while the stiff pore is absent. For the more realistic model that includes the stiff pore, significant discrepancies are observed. We identify two important aspects that need improvement in the analytical solution: the calculation of the frame stiffness moduli and the frequency dependence of attenuation and dispersion at intermediate frequencies.

\section{INTRODUCTION}

Seismic methods are widely used for detection and characterization of fluid-saturated porous rocks (Yan et al., 2019). Many studies show that a passing wave loses energy propagating through fluidsaturated rocks (Mavko and Jizba, 1991; Dvorkin et al., 1995; Pride et al., 2004; Müller et al., 2008, 2010; Gurevich et al., 2010; Pimienta et al., 2015a, 2015b). There are several phenomena responsible for energy loss associated with the pore fluid (Müller et al., 2010): for example, wave-induced fluid flow in partially saturated rocks and in fractures at the mesoscopic scale, and squirt flow in cracks or in grain-to-grain contacts at the pore scale. Rocks are heterogeneous at all scales and can be described with respect to a particular scale: the wavelength scale, the mesoscopic scale, and the pore scale. The wavelength scale obviously refers to the wavelength of a propagating wave; the mesoscopic scale is much larger than the size of individual grains and pores but smaller than the wavelength; the pore scale is of the size of individual grains, pores, and microcracks. Here, we identify fractures as discontinuities at the mesoscopic scale and cracks as discontinuities at the pore scale.

At the wavelength scale, so-called global flow occurs due to fluid pressure gradients between peaks and troughs of a passing wave (Biot, 1956, 1962). At the mesoscopic scale, fluid pressure gradients occur at scales much larger than the pore scale but smaller

Manuscript received by the Editor 13 August 2019; revised manuscript received 22 November 2019; published ahead of production 7 February 2020; published online 31 March 2020

${ }^{1}$ University of Lausanne, Institute of Earth Sciences, Lausanne, Switzerland and University of Lausanne, Swiss Geocomputing Centre, Lausanne, Switzerland. E-mail: yury.alkhimenkov@unil.ch (corresponding author); jurg.hunziker@unil.ch; beatriz.quintal@unil.ch.

${ }^{2}$ University of Lausanne, Institute of Earth Sciences, Lausanne, Switzerland. E-mail: eva.caspari@unil.ch.

${ }^{3}$ Curtin University, Department of Exploration Geophysics, Perth, Australia and CSIRO, Perth, Australia. E-mail: b.gurevich@curtin.edu.au.

${ }^{4}$ University of Geneva, Department of Earth Sciences, Geneva, Switzerland. E-mail: nicolas.barbosa@ unil.ch.

${ }^{5}$ Curtin University, Department of Exploration Geophysics, Perth, Australia. E-mail: stanislav.glubokovskikh@curtin.edu.au.

(C) 2020 Society of Exploration Geophysicists. All rights reserved. 
than the wavelength of a propagating wave: For example, flow may occur between fractures and the porous background of the rock (Brajanovski et al., 2005; Masson et al., 2006; Masson and Pride, 2007; Rubino et al., 2014; Grab et al., 2017). This wave-induced fluid flow in fractures at the mesoscopic scale can be described using the theory of Biot $(1956,1962)$ assuming heterogeneous material properties (i.e., soft, highly permeable fractures and stiff, low-permeability rock matrix). Analytical solutions for mesoscopic heterogeneities of specific geometries were developed, for example, by White et al. (1975), White (1975), Pride and Berryman (2003a, 2003b), and Pride et al. (2004).

Fluid flow in cracks at the pore scale (squirt flow) occurs between cracks and pores of different stiffness, sizes, and orientations. Such flow is believed to be significant at ultrasonic and sonic frequencies (Mavko and Nur, 1975) and also at seismic frequencies (Mavko et al., 2009; Müller et al., 2010). Several experimental studies confirmed the importance of squirt flow at different frequency ranges (Mayr and Burkhardt, 2006; Mikhaltsevitch et al., 2015; Pimienta et al., 2015a, 2015b; Subramaniyan et al., 2015; Chapman et al., 2019). An overview of early theoretical studies on squirt flow is given by Jones (1986).

Simple analytical solutions for squirt flow exist and are based on interconnected pores with different aspect ratios (O'Connell and Budiansky, 1977; Palmer and Traviolia, 1980), on the connection of a compliant crack and a stiff pore (Murphy et al., 1986; Mukerji and Mavko, 1994; Dvorkin et al., 1995; Pride et al., 2004; Gurevich et al., 2010) or on the connection of small-aspect-ratio cracks and spheroidal pores (Xu, 1998; Chapman et al., 2002; Chapman, 2003; Jakobsen and Chapman, 2009). In real rocks, examples of compliant pores are microcracks and grain contacts. The low- and high-frequency limits of the stiffness moduli in such media when saturated with a liquid are reasonably well understood (Gassmann, 1951; Mavko and Jizba, 1991; Gurevich et al., 2009b; Mavko et al., 2009). However, the frequency-dependent behavior of velocity and attenuation at intermediate frequencies is not fully understood. Numerically, squirt flow can be studied by simulating the coupled fluid-solid deformation at the pore scale, which is a difficult exercise from a computational point of view. A few numerical approaches appeared in the literature (Zhang and Toksöz, 2012; Quintal et al., 2016, 2019; Das et al., 2019). The very recent study by Das et al. (2019) simulates the coupled fluid-solid deformation at the pore scale, including inertial and nonlinear terms in the Navier-Stokes equation.

The wavelength of a passing wave is much larger than the size of individual cracks and pores; therefore, wave propagation is controlled by effective rock properties. Squirt flow is frequencydependent; thus, effective frequency-dependent stiffness moduli represent volume-averaged rock properties. This is called upscaling (going from the microscale to larger scales through averaging). We calculate the effective stiffness tensor over a representative elementary volume and analyze the corresponding stiffness moduli dispersion and attenuation. Our numerical simulation is based on the approach proposed by Quintal et al. (2016, 2019). This numerical approach describes rock matrix properties using Hooke's law and the fluid flow using the quasistatic linearized compressible Navier-Stokes momentum equation, and then, through volume averaging, calculates the effective viscoelastic stiffness tensor. We compare our numerical results with those of an analytical solution for squirt flow in anisotropic media (Collet and Gurevich, 2016).
We choose this particular analytical solution because it uses very specific material properties (e.g., pore-space geometry, elastic properties of the background medium, crack compliances, porosity, and fluid viscosity) without adjusted abstract parameters, so that the direct comparison with the numerical results is possible. The aim of this work is to (1) numerically evaluate the energy loss caused by squirt flow at the pore scale for a specific 3D pore geometry (a torus connected to a crack) and (2) compare the numerical results against a published analytical solution to examine the assumptions made during the derivation of this analytical solution. Our conclusions can also be applied to the analytical solution for squirt flow in isotropic media proposed by Gurevich et al. (2010).

The paper is organized as follows. First, we briefly describe the physics behind the squirt flow mechanism. We then describe the theory and methodology of the numerical solution. After, we briefly explain the analytical solution by Collet and Gurevich (2016). Next, we show the numerical results and the comparison with the analytical solution. Finally, we discuss the results and draw some conclusions.

\section{A BRIEF OVERVIEW OF THE PHYSICS}

Squirt flow causes seismic wave dispersion and attenuation due to the energy dissipation caused by fluid pressure diffusion at the pore scale. Assume that the pore space consists of big isometric pores and cracks with low aspect ratios, pores and cracks are fully saturated with a liquid. Cracks are much more compliant than isometric pores; therefore, a passing wave induces pressure gradients in compliant cracks. These pressure gradients force fluid to move between compliant cracks and stiff isometric pores until the pore pressure equilibrates. Due to the fluid's viscosity and associated viscous friction, this mechanism causes strong energy dissipation. Here are some useful definitions: "rock matrix" or "porous background" refer to grains and pores; "frame" refers to grains, pores, and cracks; and "modified frame" refers to grains, pores, and cracks saturated with a liquid while pores are dry.

\section{Low frequencies}

In the low-frequency regime, the fluid pressure has enough time to equilibrate during a half-cycle of the wave and become uniform throughout the pore space; therefore, Gassmann's equations are valid (Gassmann, 1951). This is called the relaxed state. Only a few parameters are needed to calculate the effective properties of the rock in the low-frequency limit: the bulk modulus of the grains, the bulk modulus of the drained frame, the bulk modulus of the fluid, and the total porosity. Then, isotropic or anisotropic Gassmann's equations can be used to calculate the effective elastic moduli of the saturated medium. Typically, the relative volume of cracks is very small (i.e., two orders of magnitude smaller compared with the volume of the stiff pores); therefore, in Gassmann's equations, only the porosity of the stiff pores can be used instead of the total porosity (Mavko and Jizba, 1991; Gurevich et al., 2010).

\section{High frequencies}

In the high-frequency regime, the fluid pressure does not equilibrate between cracks and stiff pores during a half-wave cycle. Furthermore, for cracks with very low aspect ratios saturated with a liquid, the normal crack compliance is equal to zero (but not the 
tangential compliance). Therefore, cracks become stiffer with respect to normal deformation. In other words, cracks behave as hydraulically isolated and are stiffened by the liquid. This is called unrelaxed state. The fluid in the cracks is assumed to be part of the frame material (Mavko and Jizba, 1991).

Isotropic or anisotropic Gassmann's equations can be used to calculate the effective elastic moduli of the saturated medium, but the frame moduli are stiffer compared with those at the low frequency because the normal crack compliance is negligible. Gassmann's equations are still valid because the pore pressure is uniform in all stiff pores. Thus, the effective elastic moduli are different in the high-frequency regime from those in the low-frequency regime.

\section{Intermediate frequencies}

At intermediate frequencies, a quantitative description of the physics becomes more complicated. Roughly, during the transition from a relaxed state to an unrelaxed state, the pressurized fluid stiffens the cracks as frequency increases. Therefore, this frequencydependent stiffening phenomenon also stiffens the effective stiffness moduli. This nonlinear stiffening effect is difficult to model analytically. The present numerical study sheds some light on this problem and provides a quantitative description on the frequency-dependent behavior of the moduli dispersion and attenuation.

\section{MATHEMATICAL FORMULATION}

We consider that at the pore scale, a rock is composed by a solid phase (grains) and a fluid-saturated pore space. Grains are described as a linear isotropic elastic material for which the conservation of momentum is

$$
\nabla \cdot \boldsymbol{\sigma}=0
$$

where $\boldsymbol{\sigma}$ is the stress tensor and $\nabla \cdot$ denotes the divergence operator acting on a tensor field $\boldsymbol{\sigma}$. The stress-strain relation is written as

$$
\boldsymbol{\sigma}=\mathbf{C}: \epsilon,
$$

where $\epsilon$ is the strain tensor, $\mathbf{C}$ is the fourth rank stiffness tensor, and : denotes the double dot product. For an isotropic material, the components of the stiffness tensor can be fully described by the bulk $K$ and shear $\mu$ moduli.

The fluid phase is described by the quasistatic linearized compressible Navier-Stokes momentum equation (Landau and Lifshitz, 1959):

$$
-\nabla p+\eta \nabla^{2} \mathbf{v}+\frac{1}{3} \eta \nabla(\nabla \cdot \mathbf{v})=0
$$

where $\mathbf{v}$ is the particle velocity, $p$ is the pressure, $\eta$ is the shear viscosity, and $\nabla$ denotes the nabla operator acting on vector $v$ and scalar $p$ fields. Equation 3 is valid for the laminar flow of a Newtonian fluid (i.e., low Reynolds numbers $R_{e}, R_{e}<1$ ).

\section{NUMERICAL METHODOLOGY}

For the numerical simulation, we solve the conservation of momentum equation 1 in the frequency domain for the solid and fluid phases and the generalized stress-strain relation resulting from combining equations 2 and 3 (Quintal et al., 2016, 2019). Thus, the stress-strain relation in the temporal-frequency domain is (in index form):

$$
\sigma_{i j}=\lambda e \delta_{i j}+2 \mu \epsilon_{i j}+i \omega\left(2 \eta \epsilon_{i j}-\frac{2}{3} \eta e \delta_{i j}\right),
$$

where $\epsilon_{i j}$ are the components of the strain tensor,

$$
\epsilon_{i j}=\frac{1}{2}\left(\frac{\partial u_{i}}{\partial x_{j}}+\frac{\partial u_{j}}{\partial x_{i}}\right)
$$

where $e$ is the trace of the strain tensor, $\lambda$ and $\mu$ are the Lamé parameters, $u_{i}$ denotes the displacement in the $i$ th direction, $\delta_{i j}$ is the Kronecker delta, and $\omega$ is the angular frequency.

Equations 1 and 4 are implemented into a finite-element solver. In the domain of the model representing a solid material, equation 4 reduces to Hooke's law (equation 2) by setting the shear viscosity $\eta$ to zero. Similarly, in the domain of the model representing a compressible viscous fluid, the shear modulus $\mu$ is set to zero, and hence equations 4 and 1 reduce to the linearized compressible Navier-Stokes equation (equation 3). An advantage of the proposed formulation is the natural coupling between the solid and fluid displacements at the boundaries between subdomains (Quintal et al., 2016). In our simulation, the energy dissipation is caused only by fluid pressure diffusion because inertial effects are neglected.

The whole domain is discretized using an unstructured mesh with tetrahedral elements. A direct PARDISO solver (Schenk and Gärtner, 2004) is used for solving a linear system of equations. For all models, the total number of elements ranges from $4.3 \times 10^{6}$ to $6.3 \times 10^{6}$. The total number of degrees of freedom is $17 \times 10^{6}$ or more. The simulation is performed for 13-25 different frequencies (depending on the model) from 1 to $10^{6} \mathrm{~Hz}$. For each frequency, the solver uses approximately $0.9 \mathrm{~TB}$ of RAM memory. Under a $100 \%$ performance of 32 Intel dual-socket E5-2683 v4 $2.1 \mathrm{GHz}$ cores, $2 \mathrm{~h}$ of calculations are needed for each frequency.

Direct relaxation tests are performed for numerically computing the five independent components of the effective stiffness tensor. Only five components are needed because the symmetry of the proposed geometry is transversely isotropic with the vertical axis of symmetry (VTI) (the model's geometry is explained below). For simplicity purposes, we use Voigt notation for the stress, strain, stiffness, and compliance tensors. For the normal compression test, in the vertical $z$-direction ( $C_{33}$ component), the boundary conditions are as follows: At the top boundary, a vertical displacement is assigned of the form $u_{3}=10^{-6} \times \exp (i \omega t)$; at the bottom, the vertical displacement is set to zero; at the side boundaries, the normal displacement is set to zero. For the $x y$ shear test $\left(C_{66}\right.$ component), the top boundary is assigned a displacement in $x$-direction of the form $u_{1}=10^{-6} \times \exp (i \omega t)$; at the bottom, all displacements are set to zero; at the side boundaries, displacements in $y$ - and $z$-directions are set to zero. Equivalent sets of boundary conditions are applied to obtain the relationships between the other stress and strain components and, hence, the $C_{11}, C_{44}$ components. For the $C_{13}$ component, a mixed direct test is used, the corresponding boundary conditions are given in Appendix A. The initial conditions for displacements are set to zero. The resulting stress and strains are averaged over the entire spatial domain for each frequency. Then, the complex-valued $C_{i j}(\omega)$ component is calculated for each frequency. For example, 


$$
C_{33}(\omega)=\frac{\left\langle\sigma_{3}(\omega)\right\rangle}{\left\langle\epsilon_{3}(\omega)\right\rangle},
$$

where $\langle\cdot\rangle$ represents the volume averaging over the sample volume. Equation 6 is valid because all our models exhibit VTI symmetry. We calculate the $C_{11}(\omega), C_{22}(\omega), C_{44}(\omega), C_{66}(\omega), C_{12}(\omega)=$ $C_{11}(\omega)-2 C_{66}(\omega)$ components in the same way. The inverse quality factor is calculated as (O'Connell and Budiansky, 1978)

$$
\frac{1}{Q_{i j}(\omega)}=\frac{\operatorname{Im}\left\{C_{i j}(\omega)\right\}}{\operatorname{Re}\left\{C_{i j}(\omega)\right\}} .
$$

\section{ANALYTICAL SOLUTION OF COLLET AND GUREVICH (2016)}

In this study, we compare the results of our numerical simulation against an anisotropic version of the squirt flow analytical solution of Gurevich et al. (2010) proposed by Collet and Gurevich (2016). These squirt flow solutions combine the pore pressure relaxation model of Murphy et al. (1986) with the discontinuity tensor formulation of Sayers and Kachanov (1995). Collet and Gurevich (2016) consider a double-porosity medium with aligned identical cracks embedded in a hypothetical background rock matrix made up of grains and stiff pores only (see also Pervukhina et al., 2010). This anisotropic squirt flow solution, contrary to our numerical method, assumes (A) an isotropic rock matrix embedding the cracks (we extend that assumption to a rock with a transversely isotropic background) and (B) a smaller crack aspect ratio, in the range of $10^{-3}-10^{-5}$ (we show that the crack aspect ratio can be larger).

In the Collet and Gurevich (2016) model, low- and highfrequency limits are expected to be consistent with the Gassmann and Mavko-Jizba (Mavko and Jizba, 1991) equations, respectively, and the frequency dependence is controlled by a frequencydependent effective fluid bulk modulus $K_{f}^{*}(\omega)$ of the fluid filling the crack (Gurevich et al., 2010). The crack is fully described in terms of normal and tangential compliances, $Z_{n}$ and $Z_{t}$, respectively (Kachanov, 1993; Sayers and Kachanov, 1995; Schoenberg and Sayers, 1995). They consider the so-called modified frame in which only the cracks are filled with fluid, whereas the stiffer pores are empty (Mavko and Jizba, 1991). In the low-frequency limit, the relaxed moduli of the modified frame are equal to the rock dry moduli (which means that $Z_{n}^{m f}=Z_{n}$ ); whereas in the high-frequency limit, the fluid in the cracks stiffens the frame and the unrelaxed moduli of the modified frame are equal to the dry moduli of the rock without a compliant porosity (which means that $Z_{n}^{m f}=0$, i.e., without a crack) (Mavko and Jizba, 1991; Berryman, 2007; Gurevich et al., 2009b).

In the analytical solution of Collet and Gurevich (2016), the frequency-dependent compliance tensor of the modified frame is written as (for a horizontal transversely isotropic [HTI] medium) (Kachanov, 1993; Sayers and Kachanov, 1995; Schoenberg and Sayers, 1995):

$$
S_{m n}^{M F}(\omega)=S_{m n}^{b}+\Delta S_{m n}^{M F}(\omega)
$$

where $S_{m n}^{b}$ is the compliance tensor of the rock matrix and $\Delta S_{m n}^{M F}(\omega)$ is the additional compliance due to the crack (Schoenberg and Helbig, 1997):

$$
\Delta S_{m n}^{M F}(\omega)=\left[\begin{array}{cccccc}
Z_{n}^{M F}(\omega) & 0 & 0 & 0 & 0 & 0 \\
0 & 0 & 0 & 0 & 0 & 0 \\
0 & 0 & 0 & 0 & 0 & 0 \\
0 & 0 & 0 & 0 & 0 & 0 \\
0 & 0 & 0 & 0 & Z_{t} & 0 \\
0 & 0 & 0 & 0 & 0 & Z_{t}
\end{array}\right] .
$$

The frequency-dependent normal fracture compliance is

$$
Z_{n}^{M F}(\omega)=\frac{Z_{n}}{1+\frac{Z_{n}}{\phi_{c}\left(1 / K_{f}^{*}(\omega)-\beta_{m f}\right)}},
$$

where $\phi_{c}$ is the compliant porosity (crack porosity), $Z_{n}$ is the normal compliance of the crack, and $\beta_{m f}$ is the compressibility of the modified frame (the arithmetic average). Equation 10 differs slightly from the corresponding equation in Collet and Gurevich (2016), which erroneously contained the compressibility of the grain material $\beta_{g}$ instead of $\beta_{m f}$. However, our calculation shows that the corresponding error has a very small effect on the effective properties: adding or subtracting $30 \%$ from $\beta_{m f}$ in equation 10 leads to less than $5 \%$ effect on the effective properties.

Gurevich et al. (2010) show that the stiffness of the crack can be described using a frequency-dependent fluid bulk modulus $K_{f}^{*}(\omega)$ :

$$
K_{f}^{*}(\omega)=\left[1-\frac{2 J_{1}(k a)}{k a J_{0}(k r)}\right] K_{f},
$$

where $J_{\xi}$ is Bessel function of the first kind ( $\xi=0$ or $\xi=1$ correspond to the zero- or first-order Bessel function), $K_{f}$ is the fluid bulk modulus, $a$ is the radius of the crack, and $k$ is the wavenumber of the pressure wave:

$$
k a=\frac{1}{\alpha}\left(-\frac{3 i \omega \eta}{K_{f}}\right)^{1 / 2}
$$

where $\alpha$ is the aspect ratio of the crack (thickness $h$ divided by diameter: $h /(2 a)$ ) and $\eta$ is the viscosity of the fluid. Equations 11 and 12 were obtained by imposing a zero fluid pressure boundary condition $\left(P_{f}=0\right)$ at the edge of the cylindrical crack (Gurevich et al., 2010).

The frequency-dependent stiffness tensor of the fluid-saturated medium is given by the anisotropic Gassmann's equation (Gassmann, 1951):

$$
\begin{gathered}
C_{m n}^{s a t}(\omega)=C_{m n}^{M F}(\omega)+\alpha_{m} \alpha_{n} M \\
\alpha_{m}=1-\left(\sum_{n=1}^{3} C_{m n}^{M F}\right) \beta_{g} / 3
\end{gathered}
$$

for $m=1,2,3$ and $\alpha_{4}=\alpha_{5}=\alpha_{6}=0$, and where

$$
M=\left(\varphi \beta_{f}+(1-\varphi) \beta_{g}-K^{*} \beta_{g}^{2}\right)^{-1},
$$

$$
K^{*}=\frac{1}{9} \sum_{m=1}^{3} \sum_{n=1}^{3} C_{m n}^{M F}(\omega)
$$


where $\varphi$ is the total porosity of the medium without the compliant porosity (which is neglected because the compliant porosity is two or more orders of magnitude lower than the stiff pore's porosity), $K^{*}$ is the generalized bulk modulus of the modified frame, $\beta_{f}$ is the compressibility of the fluid, $\alpha_{m}$ is the Biot-Willis coefficient, and $\beta_{g}$ is the compressibility of the grain material. The effective transversely isotropic stiffness matrix can be written for horizontal (HTI) and vertical (VTI) symmetry axis (see Appendix B). The resulting VTI stiffness matrix is used to compare the analytical solution with results from numerical simulations.

\section{RESULTS}

We model coupled solid-fluid deformation at the pore scale. In this study, we consider three 3D numerical models consisting of a pore space embedded in an elastic solid grain material. The pore spaces are

1) a flat cylinder (crack), whose edge (or tip of the crack) is subjected to zero fluid pressure boundary condition (the $P_{f}=0$ model, Figure 1a and 1b)

2) a flat cylinder whose edge is connected to a big torus (the big pore model, Figure 1c and 1d)
3) a flat cylinder whose edge is connected to a small torus (the small pore model, Figure 1e and 1f).

Topologically, a flat cylinder whose edge is connected to a big/ small torus represents one domain. The model geometry 1 with zero fluid pressure boundary condition is chosen because it represents the analytical solution of Collet and Gurevich (2016). Originally, the model geometry in 2 and 3 was proposed by Murphy et al. (1986). Then, this was modified by Gurevich et al. (2010) and Collet and Gurevich (2016) to the pore geometry in 1, where the shape of the stiff pores is irrelevant (which means that a torus is a possible choice). Each of these three 3D numerical models highlights different physical aspects of the squirt flow mechanism. The used fluid properties are those of glycerol, and the grain material has properties of quartz (Table 1). Geometric properties are shown in Table 2. As shown in Table 2, the crack diameter is $20 \mathrm{~cm}$, and the crack thickness is $0.05 \mathrm{~cm}$; therefore, the crack aspect ratio is $0.5 / 200=0.0025$. The model geometry is scalable; i.e., if all geometric parameters are divided or multiplied by any number, the numerical results will be the same. The results are controlled by the dimensionless aspect ratio.

The mesh is coarse in the solid domain, finer in the torus, and the finest in the flat cylinder representing the crack. Due to the a)

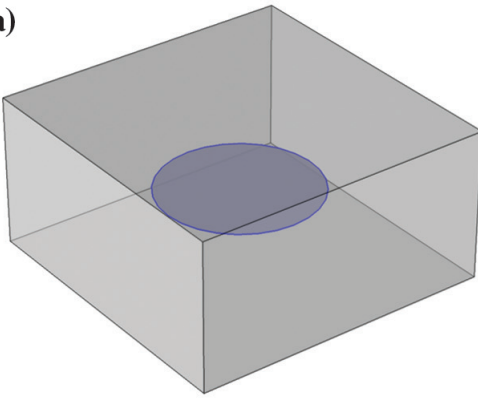

b)

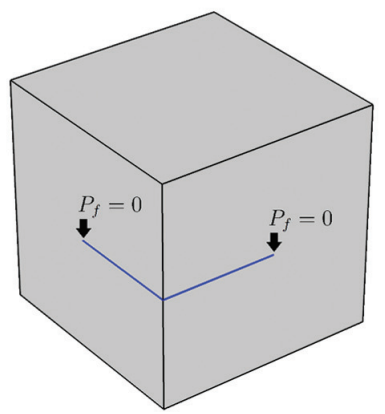

c)

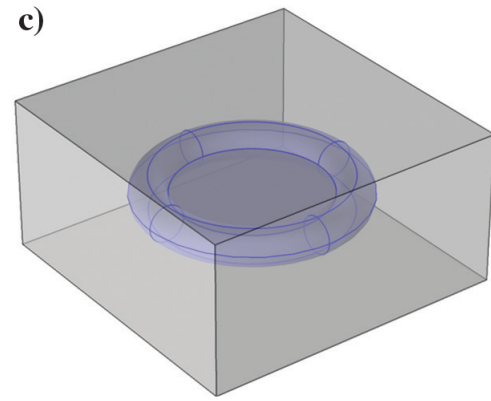

d)

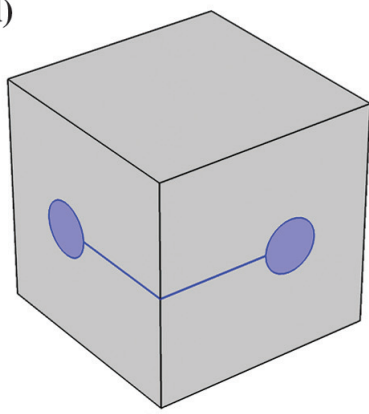

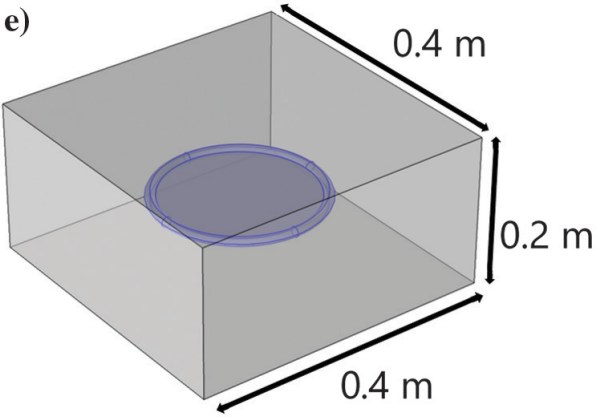

f)

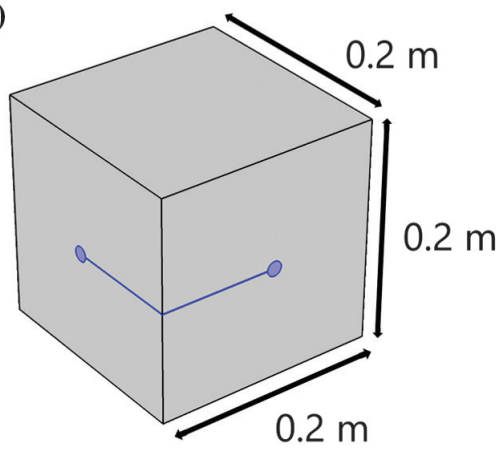<smiles>[Y]C([Y])([Y])[Y]</smiles>

Big torus

Figure 1. The $P_{f}=0$ model: (a) Sketch illustrating a flat cylinder representing a crack with fluid pressure equal to zero at the edge. The blue region represents the pore space saturated with a fluid, and the transparent gray area corresponds to the solid grain material. (b) Sketch showing a quarter of the model. Big pore model: (c) Sketch illustrating a flat cylinder representing a crack whose edge is connected to a torus representing a stiff pore. The blue region represents the pore space saturated with a fluid, the transparent gray area corresponds to the solid grain material. (d) Sketch showing a quarter of the model. Small pore model: (e) Sketch illustrating a flat cylinder representing a crack whose edge is connected to a small torus representing a stiff pore. The blue region represents the pore space saturated with a fluid, and the transparent gray area corresponds to the solid grain material. (f) Sketch showing a quarter of the model. 
model's symmetry and RAM memory limitations, the simulations are performed on a quarter of the model (Figure 1b, 1d, and 1f), a cuboid (grain material) whose size is $(0.2 \times 0.2 \times 0.2) \mathrm{m}^{3}$. In all simulations, the grain is described as a linear elastic solid material (equations 1 and 2), and the fluid is described as a compressible Newtonian fluid (equation 3).

Table 1. The material properties used for all numerical simulations.

\begin{tabular}{lll} 
Material parameter & Solid & \multicolumn{1}{c}{ Fluid } \\
\hline Bulk modulus $K$ & $36 \mathrm{GPa}$ & $4.3 \mathrm{GPa}$ \\
Shear modulus $\mu$ & $44 \mathrm{GPa}$ & $0 \mathrm{GPa}$ \\
Shear viscosity $\eta$ & $0 \mathrm{~Pa} \cdot \mathrm{s}$ & $1.414 \mathrm{~Pa} \cdot \mathrm{s}$ \\
\hline
\end{tabular}

Table 2. Geometric properties for $\boldsymbol{P}_{f}=\mathbf{0}$ model, big pore model, and small pore model. Major radius - the distance from the center of the tube to the center of the torus. Minor radius - the radius of the tube.

\begin{tabular}{llll}
\hline $\begin{array}{l}\text { Geometric } \\
\text { parameter }\end{array}$ & $\begin{array}{c}P_{f}=0 \\
\text { model }\end{array}$ & $\begin{array}{c}\text { Big pore } \\
\text { model }\end{array}$ & $\begin{array}{c}\text { Small pore } \\
\text { model }\end{array}$ \\
\hline Crack radius & $0.1 \mathrm{~m}$ & $0.1 \mathrm{~m}$ & $0.1 \mathrm{~m}$ \\
Crack thickness & $0.0005 \mathrm{~m}$ & $0.0005 \mathrm{~m}$ & $0.0005 \mathrm{~m}$ \\
Crack aspect ratio & 0.0025 & 0.0025 & 0.0025 \\
Major radius of torus & - & $0.124 \mathrm{~m}$ & $0.1067 \mathrm{~m}$ \\
Minor radius of torus & - & $0.024 \mathrm{~m}$ & $0.0067 \mathrm{~m}$ \\
Total porosity & $\approx 4.9 \times 10^{-4}$ & 0.045 & 0.0034 \\
Crack porosity & $\approx 4.9 \times 10^{-4}$ & $\approx 4.9 \times 10^{-4}$ & $\approx 4.9 \times 10^{-4}$ \\
\hline
\end{tabular}

a)

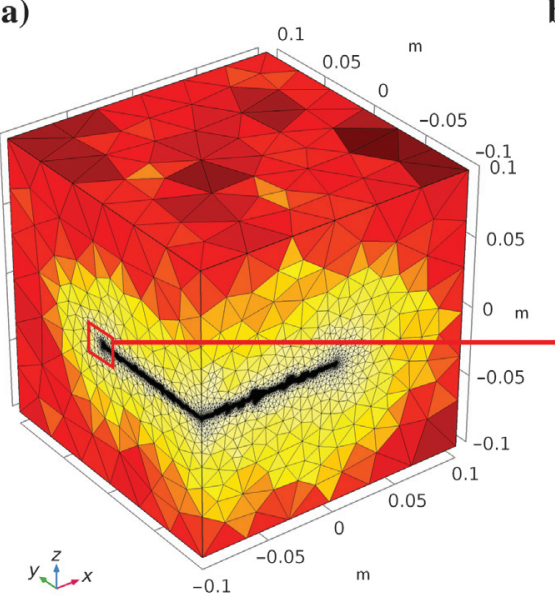

b)

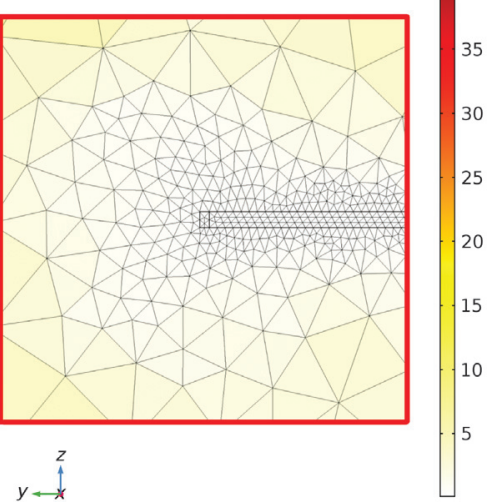

Figure 2. The element's size distribution for the $P_{f}=0$ model (Figure 1b): (a) Full model and (b) magnified part of the model showing elements in the crack (element size $0.00025-0.00015 \mathrm{~m}$ ) and in the surrounding grain material (element size 0.038$0.008 \mathrm{~m}$ ). The element's size is the smallest inside the crack and grows toward the walls of the model domain.

\section{The $\boldsymbol{P}_{f}=\mathbf{0}$ model}

\section{Numerical solution}

We consider a 3D numerical model of a flat cylinder embedded in the grain material (Figure 1a and 1b). Besides the boundary conditions applied to the external walls of the cubic model, the tip of the crack is subjected to a zero fluid pressure boundary condition $\left(P_{f}=0\right)$. This specific boundary condition corresponds to that of the analytical solution proposed by Collet and Gurevich (2016). By applying this $P_{f}=0$ boundary condition, we simulate the conditions in which the crack is filled with the liquid, whereas a virtual stiff pore acts as a sink for the fluid flow from the crack but, at the same time, has the grain material properties. The total porosity of the model is equal to the crack porosity. The proposed geometry belongs to the VTI symmetry class. Figure 2 shows the whole model domain discretization using an unstructured mesh with tetrahedral elements. The colors represent the element's size. The mesh is coarse in the grain (the elements sizes are $0.038-0.008 \mathrm{~m}$ ) and the finest in the cylinder (the elements sizes are 0.00025-0.00015 m). Because the fluid flow and, thus, the dissipation take place inside the crack, a fine, regular mesh is compulsory there, whereas in the grain material, the mesh can be much coarser. The corresponding numerical results are shown in Figure 3.

\section{Analytical solution}

To obtain the results from the anisotropic squirt flow model of Collet and Gurevich (2016), a crack embedded into an isotropic background (grain material) can be described in terms of the normal and tangential compliances of the crack $Z_{n}$ and $Z_{t}$, respectively. These parameters are calculated numerically using the following approach: The dry crack is embedded into the isotropic solid grain material, and the effective compliance tensor is calculated numerically ( $S_{\text {crack }}^{\mathrm{VTI}}$ compliance tensor). Then, we calculate the difference between the $S_{\text {crack }}^{\mathrm{VTI}}$ and the grain material $S_{\text {grain }}^{\mathrm{VTI}}$ compliance tensors. In the resulting compliance matrix, only the $S(3,3), S(4,4)$, and $S(5,5)$ components are nonnegligible. Thus, $Z_{n}=S(3,3)$ and $Z_{t}=S(4,4)=S(5,5)$. Using equations 8-16, the effective stiffness tensor is calculated, which then is transformed from HTI to VTI symmetry.

\section{Comparison}

Only the results for the $C_{33}$ component are shown in Figure 3 because the other components are constant with frequency, except $C_{13}$, which varies slightly. The analytical and numerical results are in a very good agreement including the low- and high-frequency limits, the frequency dependence of the dispersion and attenuation curves at intermediate frequencies, and the left and right asymptotes of the attenuation curve (Figure 3). This comparison shows that our numerical result is correct and can be used to simulate more complicated models. Furthermore, such a good agreement indicates that our numerical solution fulfills conditions $\mathrm{A}$ and $\mathrm{B}$ of the analytical solution.

Figure 4 shows snapshots of the fluid pressure $P_{f}$ in the crack at three different frequencies. In 
the relaxed state, there is enough time for pressure equilibration between the crack and zero fluid pressure at the crack tip (because of the $P_{f}=0$ boundary condition) (Figure 4, LF). Therefore, the fluid in the crack does not impose any stiffening to the crack. The stiffness of the crack is the same as if it was dry (thus, the $P_{f}=0$ model is not consistent with Gassmann equations in the low-frequency limit). At intermediate frequencies, there is a large pressure gradient in the crack, which corresponds to the maximum attenuation (Figure 4, FC). In the unrelaxed state, there is no equilibration between the fluid pressure inside the crack and zero fluid pressure at the tip of the crack (Figure 4, HF). Therefore, the crack behaves as hydraulically isolated and the fluid highly stiffens the crack, so that the normal compliance of the crack approaches zero (tangential compliance is not zero).

\section{Big pore model}

\section{Numerical solution}

We consider a 3D numerical model of a flat cylinder whose edge is connected with a big torus (Figure 1c and 1d). The flat cylinder

a)
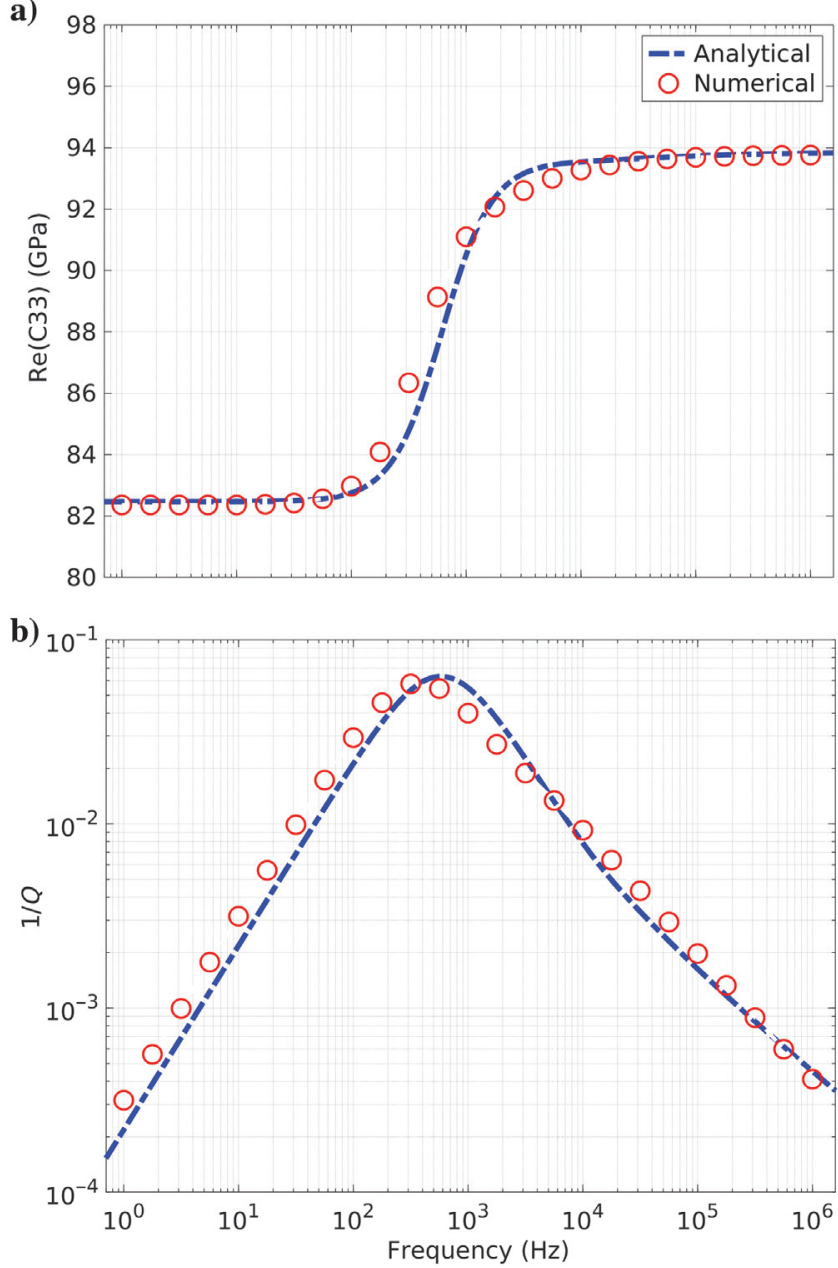

Figure 3. Numerical and analytical results for the model with fluid pressure equal to zero at the edge of the crack $\left(P_{f}=0\right)$ (Figure 1a and 1b): (a) Real part of the $C_{33}$ component and (b) dimensionless attenuation for the $C_{33}$ component. Each red circle corresponds to a numerical calculation. representing a crack has the same radius and thickness as in the $P_{f}=0$ model. The torus represents a much stiffer pore. The torus and the crack are embedded into a cuboid of grain material and are fully saturated with a liquid. The proposed geometry belongs to the VTI symmetry class. Numerical results for the $C_{m n}$ components using five direct tests are shown in Figure 5. From this figure, only the $C_{33}$ component seems to be frequency-dependent, but the $C_{13}$ component is also slightly frequency dependent.

\section{Extended analytical solution}

For the comparison between the analytical solution and the numerical results, all stiffness properties of the dry medium are calculated numerically (or are the same as in the numerical simulation) and used as the input to the analytical solution. To obtain the corresponding results from the analytical solution, normal $Z_{n}$ and

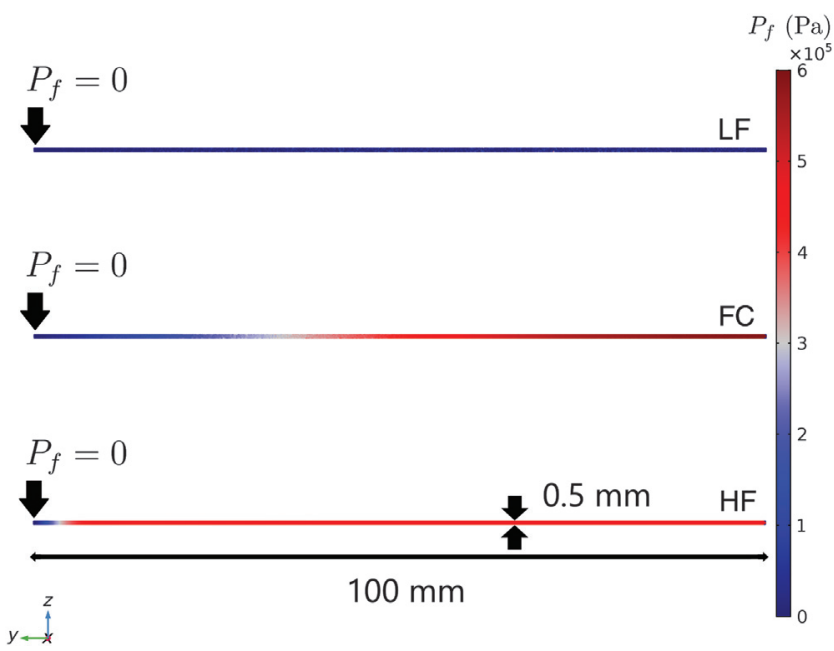

Figure 4. Snapshots of the fluid pressure $P_{f}$ in the fracture at three different frequencies for the $P_{f}=0$ model: LF - low frequency (relaxed state), FC - intermediate frequency (close to the characteristic frequency), and $\mathrm{HF}$ - high frequency (unrelaxed state).

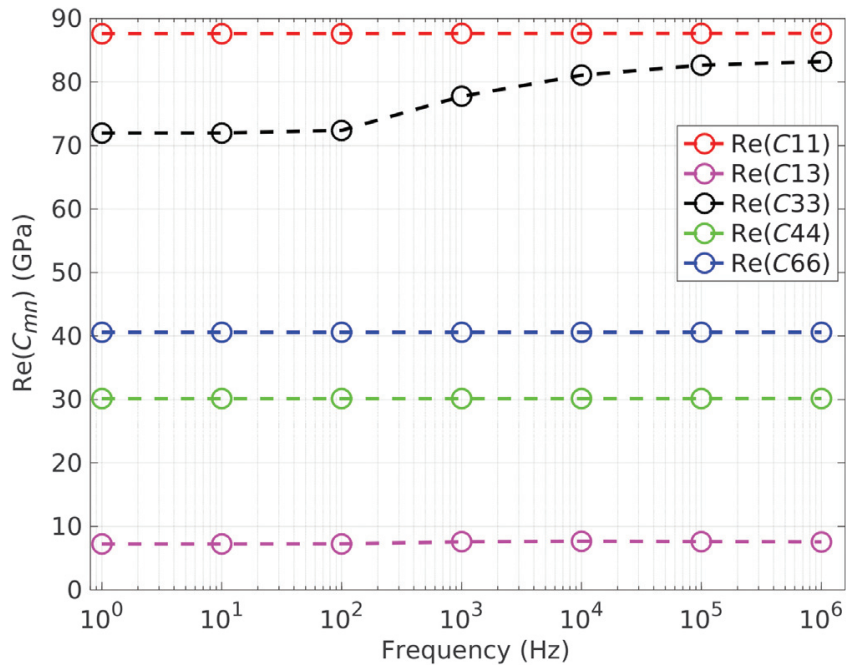

Figure 5. Numerical results for the real part of $C_{m n}$ components for the big pore model (Figure 1c and 1d) using five direct tests. 
tangential $Z_{t}$ compliances of the crack are needed. To obtain $Z_{n}$ and $Z_{t}$, we numerically calculate several (homogenized) elastic stiffness tensors of a dry medium (Figure 6): a torus embedded into the solid grain material ( $C_{1}^{\mathrm{VTI}}$ stiffness tensor), a crack embedded into a medium described by the $C_{1}^{\mathrm{VTI}}$ stiffness tensor ( $C_{2}^{\mathrm{VTI}}$ stiffness tensor), and a torus connected with a crack embedded into an isotropic solid grain material ( $C_{3}^{\mathrm{VTI}}$ stiffness tensor). Then, all of the $C^{\mathrm{VTI}}$ stiffness tensors are inverted to the corresponding compliance tensors $S^{\mathrm{VTI}}$. For obtaining $Z_{n}$ and $Z_{t}$, there are at least two possible workflows.

- Workflow A: We calculate $Z_{n}$ and $Z_{t}$ using the difference between the $S_{1}^{\mathrm{VTI}}$ compliance tensor and the $S_{2}^{\mathrm{VTI}}$ compliance tensor (Figure 6). In this case, we first homogenize the torus (and obtain $C_{1}^{\mathrm{VTI}}$ ) and then embed the crack into this homogenized material $C_{1}^{\mathrm{VTI}}$. Thus, $Z_{n}$ and $Z_{t}$ do not account for the fact that the crack is connected with the stiffer pore, which implies a different geometry from the one shown in Figure $1 \mathrm{c}$ and $1 \mathrm{~d}$ for calculating the stiffness tensor of the dry material. This approach is used by Collet and Gurevich (2016).

- Workflow B: We calculate the normal and tangential compliances using the difference between the $S_{1}^{\mathrm{VTI}}$ compliance tensor and the $S_{3}^{\mathrm{VTI}}$ compliance tensor (Figure 6). In this case, we also first homogenize the torus but then embed the crack connected to the torus into the solid grain material. Thus, the $C_{3}^{\mathrm{VTI}}$ stiffness tensor corresponds to the dry stiffness tensor of the model, so the difference $S_{1}^{\mathrm{VTI}}-S_{3}^{\mathrm{VTI}}$ gives the correct compliances $Z_{n}$ and $Z_{t}$ for the dry model (using the homogenized material $C_{1}^{\mathrm{VTI}}$ ). These compliances $Z_{n}$ and $Z_{t}$ approximately correspond to the crack embedded into the $C_{1}^{\text {VTI }}$ material and have a total effective radius equal to the radius of the crack itself plus the additional minor diameter of the torus. This approach is similar to the one used in Gurevich et al. (2009b).

The generalization of Collet and Gurevich (2016) to an anisotropic porous background is straightforward because the principal

Workflow A

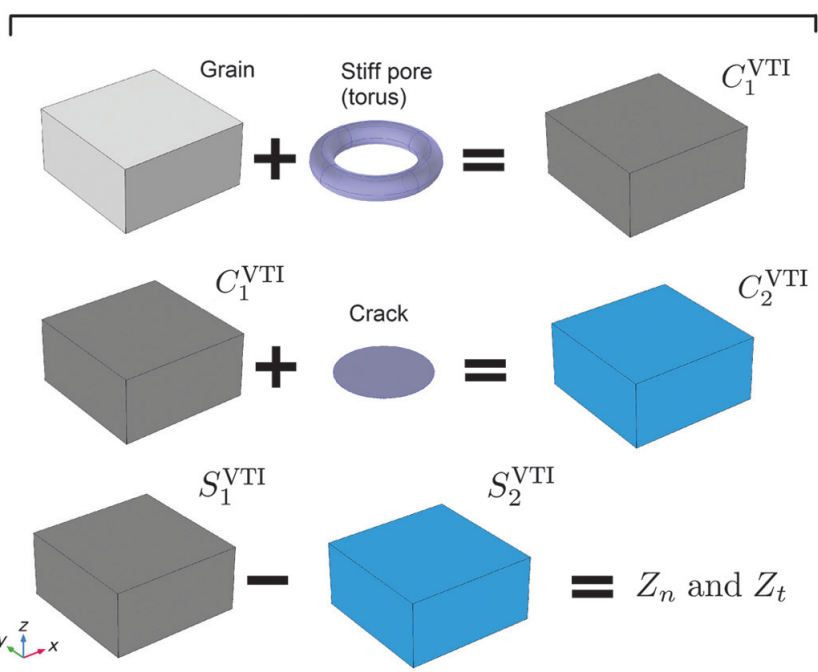

symmetry axis of the torus (stiff pore), embedded into the grain material is the same as that of the crack and the torus embedded into the grain material, and the corresponding frame stiffnesses are described by the same equations 8 and 9 . Thus, we are able to fulfill condition A for anisotropy of the rock matrix. Moreover, according to Collet and Gurevich (2016), the assumption $Z_{n}^{m f}=0$ in the highfrequency limit holds only for cracks with aspect ratios lower than 0.001 . However, we have already seen in our numerical results in Figure 3 (for an aspect ratio of 0.0025) that this assumption also holds for cracks with larger aspect ratios (up to 0.005). Therefore, we conclude that our extended numerical simulation fulfills conditions $\mathrm{A}$ and $\mathrm{B}$ of the analytical solution of Collet and Gurevich (2016).

Using equations 8-16, the complex-valued stiffness tensor is calculated for workflows $\mathrm{A}$ and $\mathrm{B}$. The difference between these two solutions is due to the different $Z_{n}$ and $Z_{t}$ parameters that are used in equations $8-16$.

\section{Comparison}

Figure 7 shows results for the $C_{33}$ complex-valued component of the stiffness tensor obtained from the numerical simulation and from the analytical solution with two different sets of normal and tangential compliances derived from workflows A and B (Figure 6). The dispersion curves show that in the high-frequency limit, the two analytical solutions and the numerical result are in reasonably good agreement. In the low-frequency regime, the dispersion curve predicted by the analytical result with workflow A is much stiffer compared to the numerical result. This is because the calculation of $Z_{n}$ and $Z_{t}$ involved the strong assumptions described above. The attenuation curve from workflow A shows, only by chance, a good match with the numerical result except for the asymptotic behavior in the high-frequency regime. In the low-frequency regime, the analytical result with workflow B is close to the numerical dispersion curve: a difference of approximately $2 \mathrm{GPa}$ because parameters $Z_{n}$ and $Z_{t}$ cannot fully describe the elastic behavior of a crack. In this

Figure 6. Sketch illustrating the calculation of normal and tangential compliances of the crack for workflows A and B. The term $S_{r}^{\mathrm{VTI}}$ denotes the compliance tensor, which is the inverse of the corresponding stiffness tensor. That is, $S_{r}^{\mathrm{VTI}}=\left(C_{r}^{\mathrm{VTI}}\right)^{-1}$, for $r=1,2,3$. The resulting $Z_{n}$ and $Z_{t}$ are used to calculate the analytical solution for corresponding models (Figure 1). 
case, the $C_{13}$ component is affected and causes this difference in the anisotropic Gassmann's equations. The attenuation curve predicted by the analytical solution with workflow B is different from the numerical result: The characteristic frequency is shifted to the left, and the maximum attenuation is approximately twice that of the numerical results.

Figure 8 shows snapshots of the fluid pressure $P_{f}$ in the fracture and the stiff pore at three different frequencies. In the relaxed state, the fluid pressure is equilibrated throughout the pore domain (Figure 8, LF). At intermediate frequencies, there is a large pressure gradient in the crack, which corresponds to the maximum attenuation (Figure 8, FC). In the unrelaxed state, there is no pressure equilibration between the fluid pressure inside the crack and the fluid pressure inside the torus (Figure $8, \mathrm{HF}$ ). In the relaxed state (Figure $8, \mathrm{LF}$ ), the pore pressure inside the stiff pore is slightly increased compared to the unrelaxed state (Figure 8, HF). That is due to a finite volume of the stiff pore. Although the crack porosity is several orders of magnitude lower than the stiff pore porosity, such a

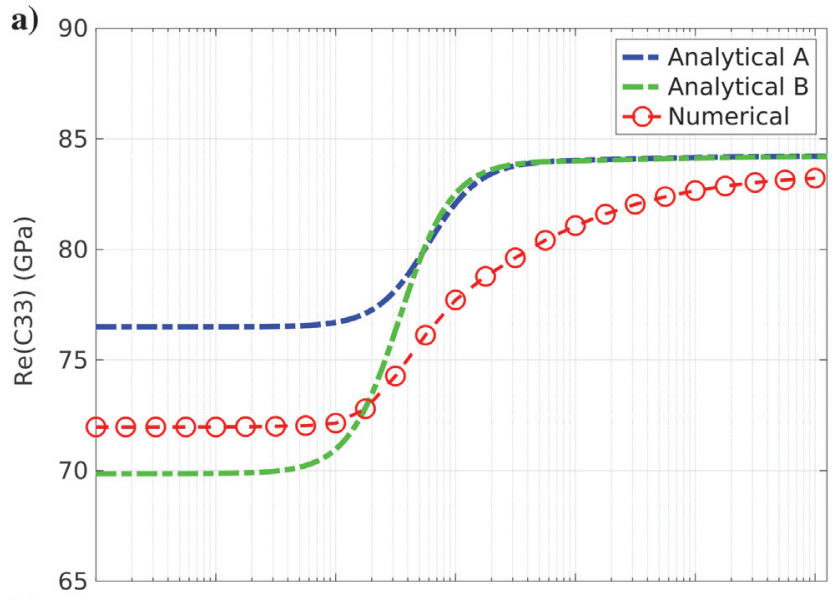

b)

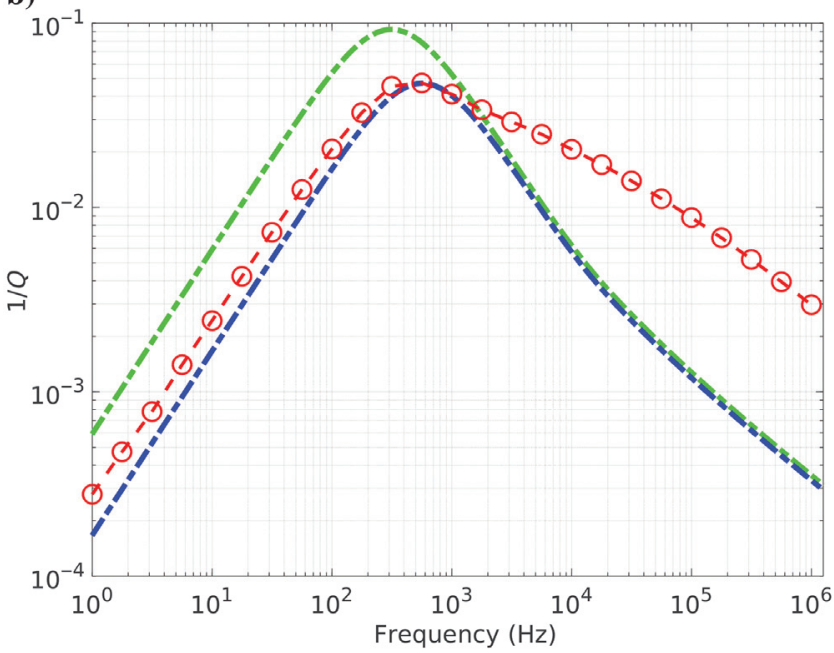

Figure 7. Numerical and analytical results for the big pore model (Figure 1c and 1d): (a) Real part of the $C_{33}$ component and (b) dimensionless attenuation for the $C_{33}$ component. Each red circle corresponds to one test. The maximum of the attenuation curve (analytical solution with workflow A, the blue curve) is exactly the same as in the numerical result by a chance because the comparison between the analytical solution and numerical result for the modified frame exhibit discrepancy; it is explained in the text. small volume of fluid still slightly increases the pore pressure inside the stiff pore in the relaxed state.

Few numerical artifacts (the red points) can be seen in the torus in the low and intermediate frequencies (Figure 8, LF and FC); this is due to the fact that the mesh is not very fine inside the torus. Due to RAM memory limitations, it is difficult to significantly increase the resolution inside the torus, but the simulation has been run for several different mesh distributions and element sizes converging to the same output results. The limits of the dispersion curve were also verified: The low-frequency limit was verified by running dry elastic simulations and adding fluid using the Gassmann's equations, and the highfrequency limit was verified by running elastic simulations and thus representing fluid as an elastic material. The Kramers-Kronig relation was also used to verify the consistency of the numerical results: The attenuation curve was reproduced from the dispersion curve using the Kramers-Kronig relation. Thus, we conclude that these artifacts are local and do not affect our results and our results are accurate. There are also some small boundary effects resulting in minor artifacts in the crack (artifacts appear near the walls of the cube), which have negligible effects on our numerical results. The snapshots of fluid pressure are from a diagonal slice in the $x y$-plane to avoid boundary effects.

\section{Small pore model}

\section{Numerical solution}

We consider the same model as in the previous section (the big pore model), but now the volume of the torus is smaller (Figure 1e and 1f). The flat cylinder (representing a crack) has the same radius and thickness as in the $P_{f}=0$ and big pore models. The torus and the crack are embedded into a cuboid of grain material. The proposed geometry belongs to the VTI symmetry class. Our numerical results for the $C_{33}$ component are shown in Figure 9.

\section{Extended analytical solution}

To obtain the results from the analytical solution, $Z_{n}$ and $Z_{t}$ are calculated using the same extended analytical solution as in the big

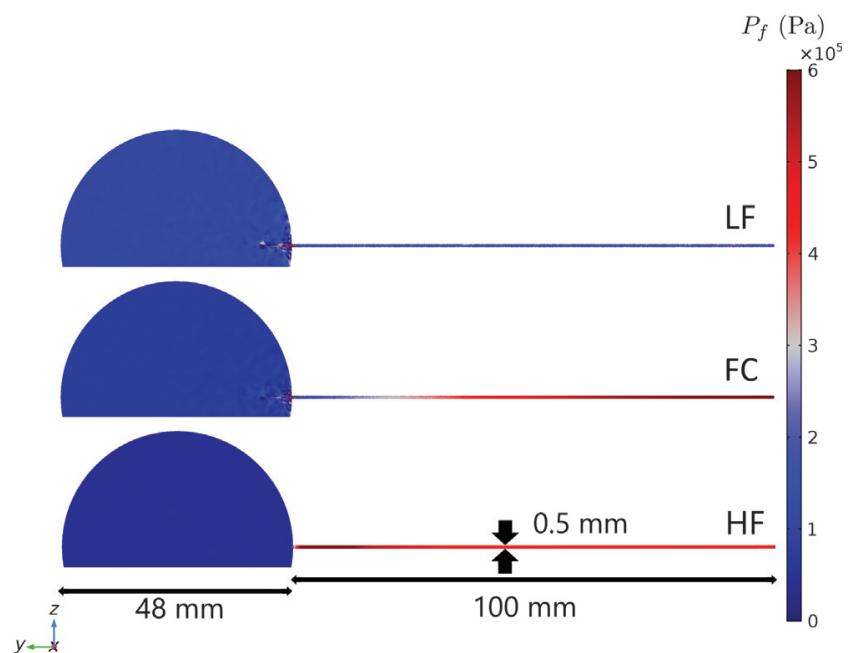

Figure 8. Snapshots of the fluid pressure $P_{f}$ in the fracture at three different frequencies for the big pore model: LF - low frequency (relaxed state), FC - intermediate frequency (close to the characteristic frequency), and $\mathrm{HF}$ - high frequency (unrelaxed state). 
pore model. Then, using equations $8-16$, the complex-valued stiffness tensor is calculated for the output of workflows A and B (Figure 6).

\section{Comparison}

Figure 9 shows the results for the $C_{33}$ complex-valued component of the stiffness tensor obtained from the numerical simulations and from the analytical solution with two different sets of normal and tangential compliances derived from workflows A and B. The dispersion and attenuation are small in this model; therefore, the two analytical solutions show an apparently better agreement than for the big pore model (Figure 7). However, the relative difference between the analytical and numerical results is comparable to those for the big pore model. Here, both analytical solutions predict much higher attenuation compared to the numerical result.

Figure 10 shows snapshots of the fluid pressure $P_{f}$ in the fracture and in the stiff pore at three different frequencies. The only geometric difference between this small pore model and the big pore model is that the torus is smaller. The volume of the small torus is only approximately seven times bigger than the crack volume; therefore,
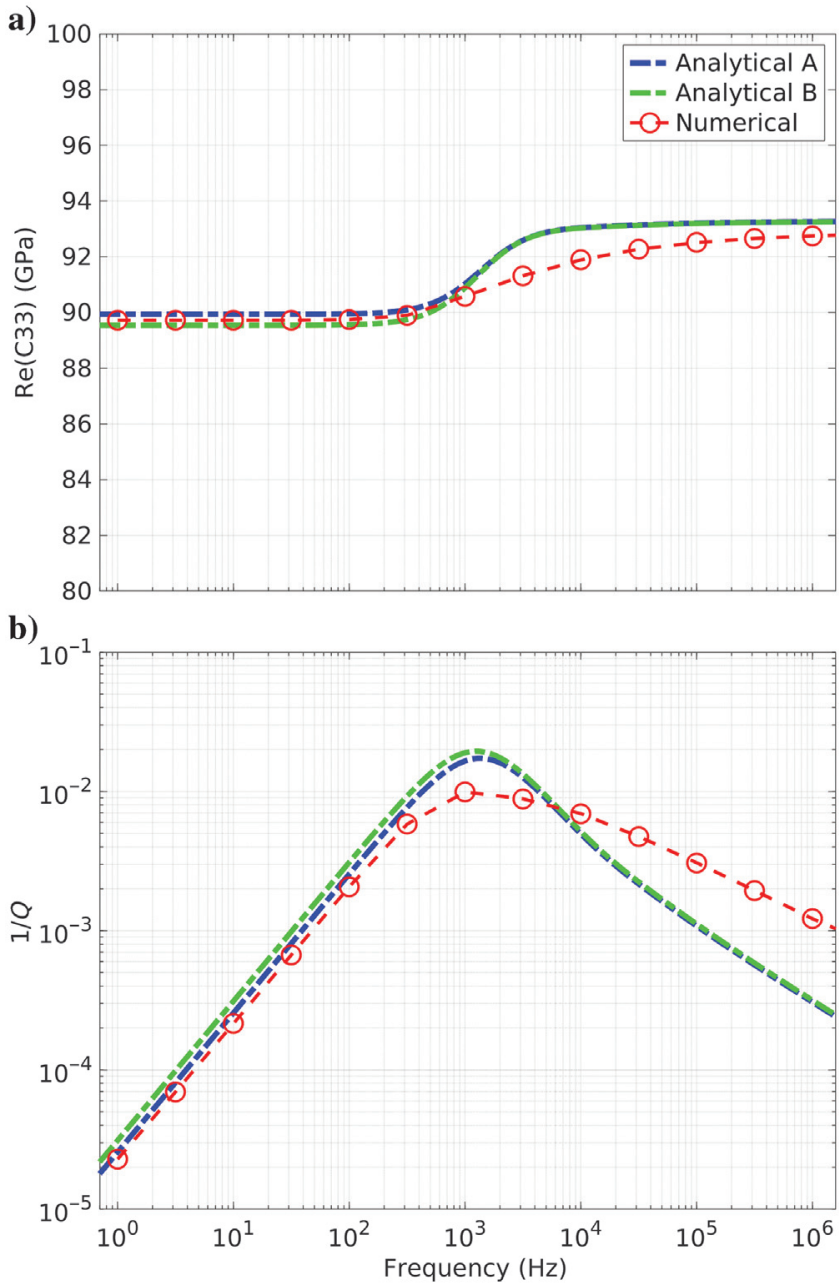

Figure 9. Numerical and analytical results for the small pore model (Figure 1e and 1f): (a) Real part of the $C_{33}$ component and (b) dimensionless attenuation for the $C_{33}$ component. the fluid pressure gradient equilibrates faster, and a larger value of the fluid pressure is observed in the relaxed state than that in the big pore model (Figure 8, LF). The characteristic frequency is higher, and the overall dissipation is lower. The numerical artifacts observed in these snapshots have negligible effect on our numerical solution as explained above.

\section{Modified frame}

As mentioned before, the modified frame is a virtual rock, in which only the crack is filled with fluid, whereas the stiffer pore is empty (Mavko and Jizba, 1991). In the low-frequency limit, the relaxed moduli of the modified frame are equal to the rock dry moduli. In the high-frequency limit, the fluid-saturated crack stiffens the frame, and the unrelaxed moduli of the modified frame are equal to the dry moduli of the rock without a compliant porosity (i.e., without a crack).

Considering the big pore model (Figure 1c and 1d), the torus is dry, whereas the crack is filled with a compressible Newtonian fluid (glycerol). This configuration corresponds to the modified frame. Because the torus is dry, this configuration implies $P_{f}=0$ boundary condition at the edge of the crack. The main difference between this modified frame model and the $P_{f}=0$ model (Figure 1a and $1 \mathrm{~b}$ ) is the actual presence of the torus, which affects the effective properties of the model.

To compare the numerical results with the analytical solution, $Z_{n}$ and $Z_{t}$ are calculated for workflows $\mathrm{A}$ and $\mathrm{B}$. Then, using equations 8-13, the complex-valued stiffness tensor $C_{m n}^{M F}(\omega)$ is calculated. In these analytical solutions, we do not use the Gassmann equation to saturate the medium because the stiff pore is dry (equation 13 becomes $C_{m n}^{\text {sat }}(\omega)=C_{m n}^{M F}(\omega)$ ). Figure 11 shows results for the $C_{33}$ complex-valued component of the stiffness tensor obtained from the numerical result and from the analytical solution with two different sets of normal and tangential compliances derived from workflows A and B. The dispersion curves show that in the highfrequency limit, the two analytical and the numerical solutions are in a very good agreement, and the analytical solution with workflow $\mathrm{B}$ also shows a good fit in the low-frequency regime. However, the

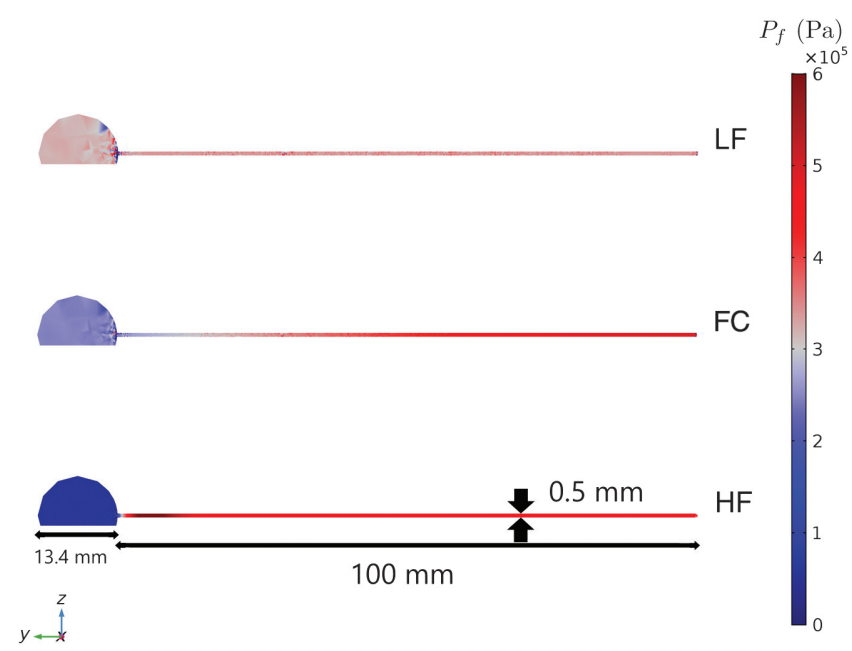

Figure 10. Snapshots of the fluid pressure $P$ in the fracture at three different frequencies for the small pore model: LF - low frequency (relaxed state), FC - intermediate frequency (close to the characteristic frequency), and $\mathrm{HF}$ - high frequency (unrelaxed state). 
frequency dependences of the dispersion and attenuation curves obtained via the numerical simulation have a completely different shape compared to both analytical solutions. More specifically, the maximum amplitude of the attenuation peak and the right slope of the attenuation curve are different compared to both analytical solutions. The previous very good fit of the attenuation magnitude between the analytical solution with workflow A and the numerical result for the saturated case (Figure 7) is coincidental. This becomes apparent by the mismatch of the attenuation curves for the corresponding modified frame between the analytical solution with workflow $\mathrm{A}$ and the numerical solution shown in Figure 11. In Figure 7, this discrepancy was coincidentally corrected by applying the anisotropic Gassmann equation to the analytical model with workflow A.

\section{Comparison to full saturation}

Figure 12 shows numerical results for four models: the modified frame model for the big pore model, the corresponding fully saturated big pore model, the modified frame model for the small pore model, and the corresponding fully saturated small pore model.
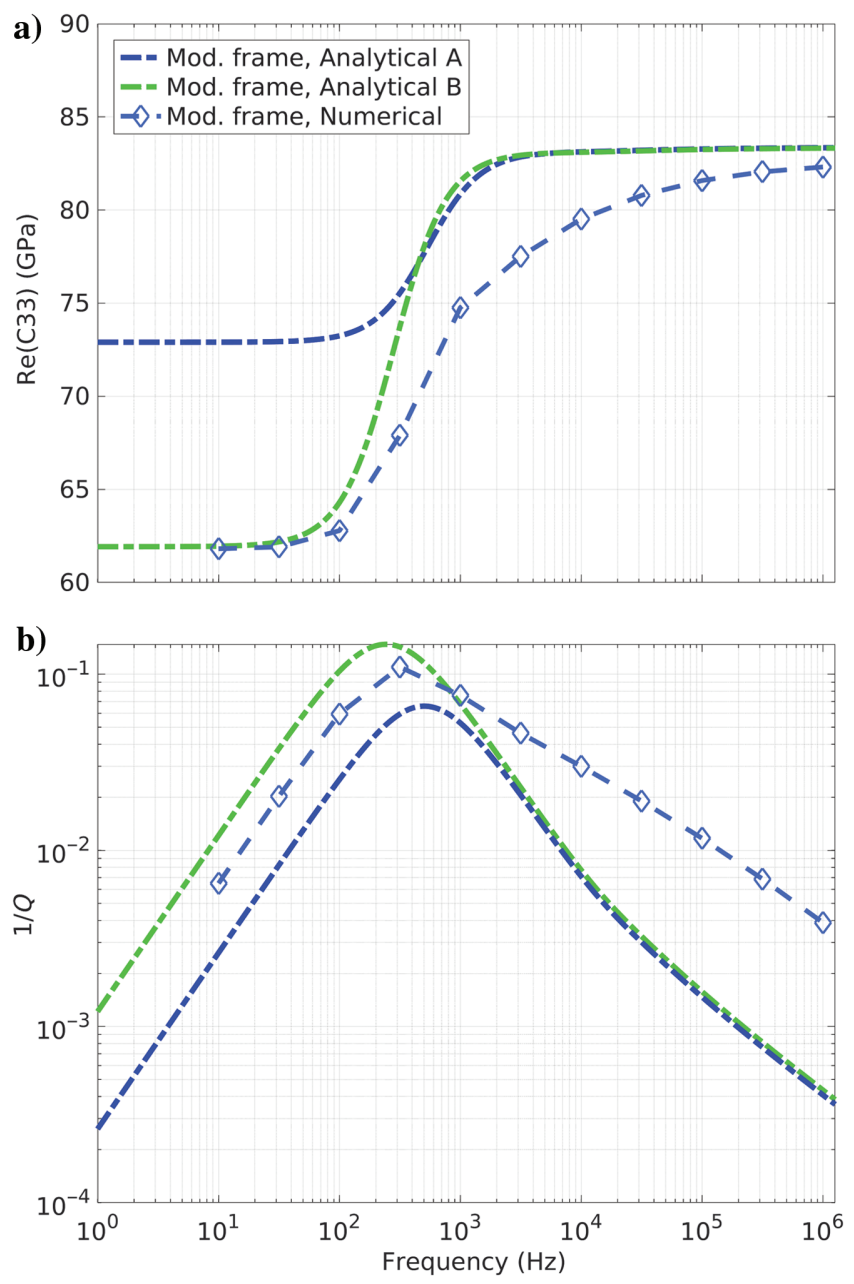

Figure 11. Numerical and analytical results for the modified frame big pore model: (a) Real part of the $C_{33}$ component and (b) dimensionless attenuation for the $C_{33}$ component. Each rhombus corresponds to a numerical calculation. Crack aspect ratio is 0.0025 .
Basically, the difference between the dashed and solid curves is due to the presence of the fluid in a big or small torus. The right slope of the attenuation curves of the modified frame models is approximately the same independently of the size of the torus. The same behavior follows for the saturated cases: the right slope of the attenuation curves of saturated models is approximately the same independently of the size of the torus.

\section{Numerical results for different crack aspect ratios}

We consider the fully saturated big pore model and change the aspect ratio of the crack. In other words, we keep the same length of the crack but increase its aperture. Figure 13 shows results for the $C_{33}$ complex-valued component of the stiffness tensor obtained from the numerical simulation with aspect ratios of 0.0025 , 0.005 , and 0.01 . For comparison, we also show results for the $C_{33}$ complex-valued component of the $P_{f}=0$ model. According to Figure 13, the frequency dependence of the dispersion and attenuation curves for those three aspect ratios is the same. The shift in the characteristic frequency is controlled by the aspect ratio of
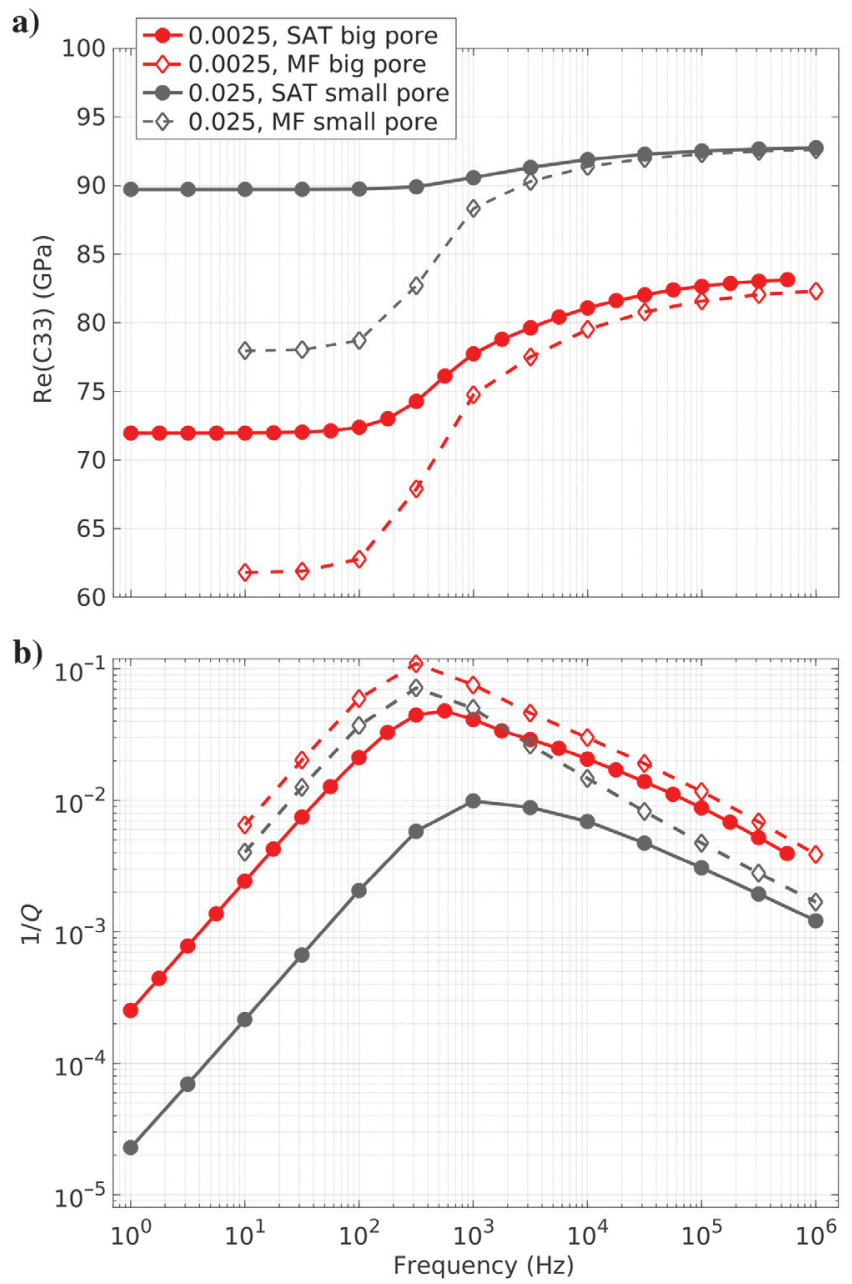

Figure 12. Numerical results and the modified frame big pore, the modified frame small pore models and corresponding saturated models: (a) Real part of the $C_{33}$ component and (b) dimensionless attenuation for the $C_{33}$ component. Each red circle or rhombus corresponds to a numerical calculation. 
the crack. The attenuation magnitude is the same at those three characteristic frequencies.

\section{DISCUSSION}

There are two main sources of strong discrepancies between the numerical results and the analytical solution. First, the calculation of the frame stiffness moduli in the analytical solution considers the pores and cracks as being disconnected. Second, the frequency dependence of attenuation and dispersion at intermediate frequencies in the analytical solution is based on several strong assumptions and shows significant discrepancies compared to the numerical results. The first one could be improved in the current analytical squirt flow solutions by using a more complete approach for calculating the frame moduli. The second one is more difficult and unclear how to implement via an analytical solution. Another observation from this study is the frequency dependence of the $C_{13}$ component of the frame moduli, which is difficult to take into account in the analytical solution, but, in general, the effect is of minor importance.
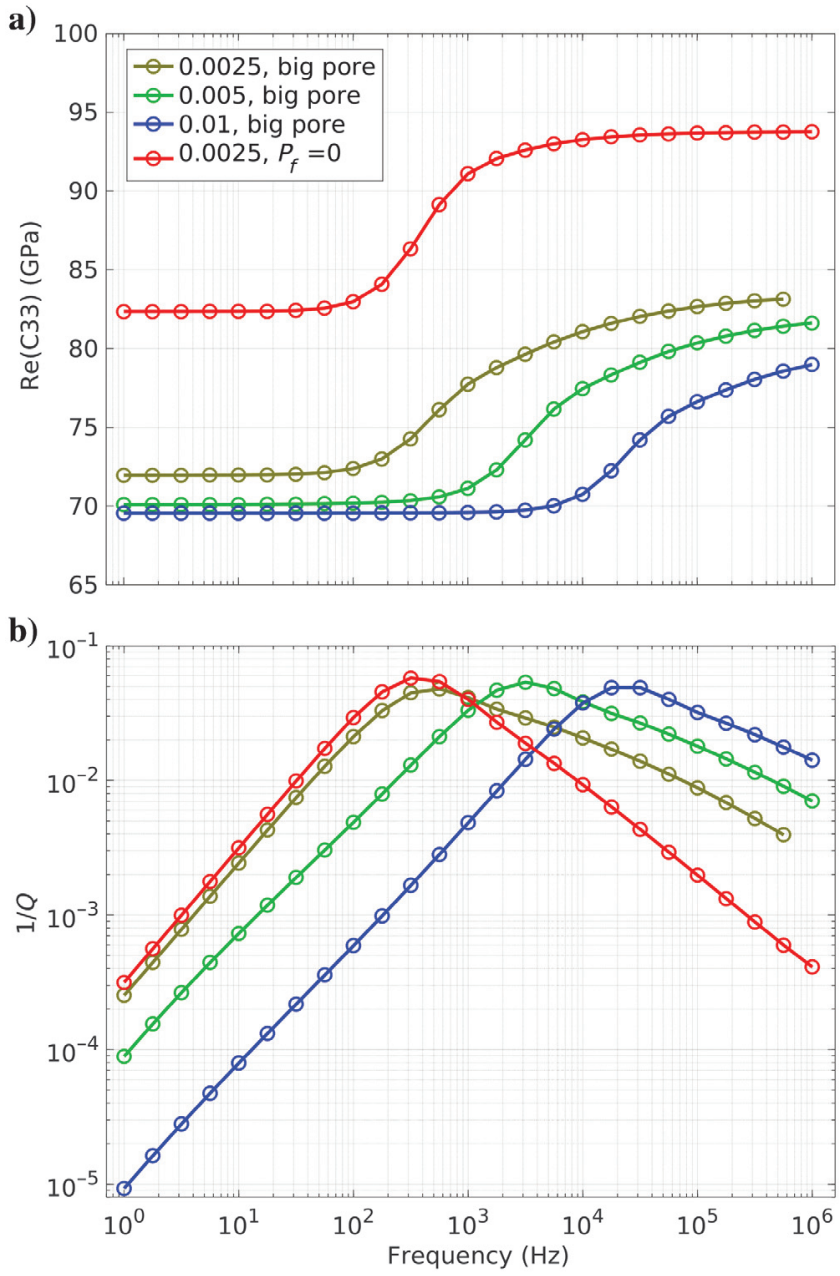

Figure 13. Numerical results for the big pore model (Figure 1c and $1 \mathrm{~d}$ ) and the $P_{f}=0$ model (with fluid pressure equal to zero at the edge of the cylinder) (Figure 1a and 1b), cracks with different aspect ratios of $0.0025,0.005$, and 0.01: (a) Real part of the $C_{33}$ component and (b) dimensionless attenuation for the $C_{33}$ component.
We showed that the analytical solution of Collet and Gurevich (2016) is accurate for the $P_{f}=0$ model where the stiff porosity is zero, but it does not accurately approximate the results for models with nonzero stiff porosity. Despite all of the discrepancies between the analytical solution and the numerical results, this analytical solution is probably the best for such an analysis because it uses specific material and geometric properties without any fitting parameters.

\section{Design and calculation of the modified frame and dry frame moduli}

One of the most important outcomes of this numerical study is the adequate calculation of the double-porosity frame moduli. By double porosity, we mean that the pore space consists of two types of pores: the stiff isometric pore and the compliant crack. Furthermore, the pore and the crack are connected. The analytical solution by Collet and Gurevich (2016) is based on the two-step homogenization approach: They consider a rock matrix (grains and a pore) and then embed a crack into that rock matrix using the normal and tangential compliances of the crack. This corresponds to our workflow A (Figure 6). Roughly, this workflow gives the frame moduli accounting for the crack and the stiff pore as being not connected (i.e., as if the crack and the pore are embedded simultaneously into the grain material but disconnected and far from each other, so the elastic interactions are very small; see Figure 14). Hence, this workflow significantly overestimates the stiffness of the frame material and significantly underestimates dispersion and the overall attenuation (Figure 7). In this case, there is a controversial issue: Gurevich et al. (2010) and Collet and Gurevich (2016) study the effect of fluid flow between the crack and the stiff pore and, at the same time, they use the frame moduli of disconnected cracks and pores. Workflow B shows a better prediction of the low- and high-frequency limits of the dispersion curve and the overall attenuation but significantly overestimates the maximum attenuation (due to amplification of the imaginary part of $Z_{n}^{M F}(\omega)$ if we connect the crack and the stiff pore). Quantitatively, $Z_{n}$ in workflow B is

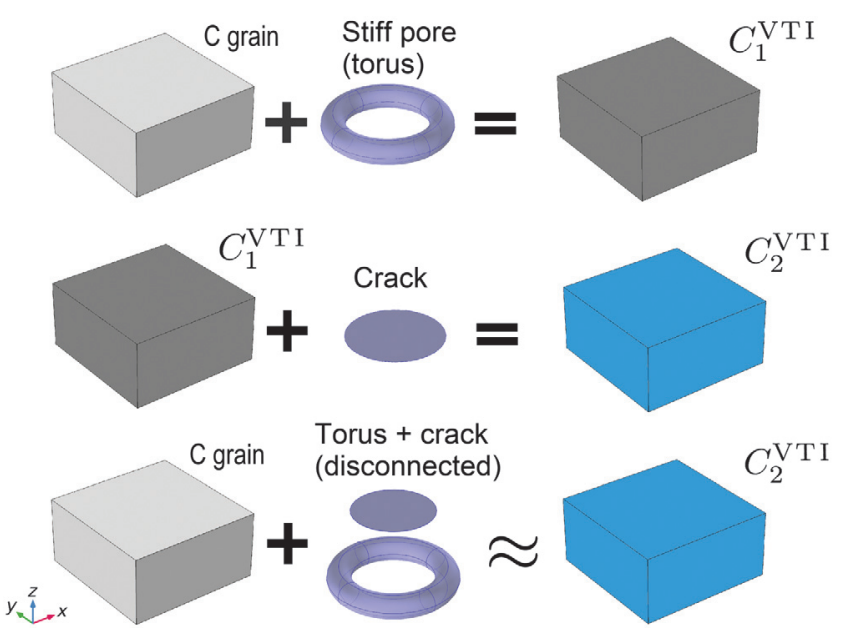

Figure 14. Sketch illustrating that the two-step homogenization approach produces the $C_{2}^{\mathrm{VTI}}$ stiffness tensor, which is approximately equal to the case if we embed the crack and the stiff pore (disconnected) into the grain material. 
two times larger than $Z_{n}$ in workflow A, which is only due to the connectivity effect of the crack and the pore. Thus, any change in the geometry of the pore space will immediately affect the stiffness of the rock and the fluid-flow properties; thus these properties are strongly coupled.

The two-step homogenization approach for calculating the effective elastic properties of cracked-porous rocks can be used considering a mesoscopic scenario. In this scenario, the cracks are at least two orders of magnitude larger than the pores; therefore, the two-step homogenization approach might work quite well (i.e., the torus connected to the crack makes no sense at a mesoscopic scale because cracks or fractures are much larger than the pores). Such double-porosity media with mesoscopic cracks were studied by Galvin and Gurevich (2009), Gurevich et al. (2009a), and Guo et al. (2017a, 2017b).

Collet and Gurevich (2016) assume that the crack can be accurately described by the two parameters: normal and tangential compliances $Z_{n}$ and $Z_{t}$ (however, we show that the $C_{13}$ component is also different). This approach is known as the linear-slip theory (Schoenberg and Sayers, 1995). There are several solutions that link the crack compliances $Z_{n}$ and $Z_{t}$ with crack geometry and the stiffness of the background medium (Walsh, 1965; Kachanov, 1993). Some insights into these theories are given by Bakulin et al. (2000a, 2000b). Note that the linear-slip theory leads to the socalled noninteractive approximation (Sevostianov and Kachanov, 1999; Kachanov and Sevostianov, 2018), which assumes that different cracks (or cracks and surrounding pores) do not interact with each other; for higher porosity - more complicated effective methods should be used as shown in the numerical study by Saenger et al. (2004).

The effect of connectivity of the pore space is usually not taken into account properly in analytical solutions due to theoretical difficulties (as far as we know, there is no general analytical solution of this problem) and due to the fact that the information about the connectivity of the pore space in real rocks is usually unknown. However, there are several solutions to this problem, for example, by introducing an additional "pore space connectivity" parameter. This parameter can be inverted by linking the effective properties predicted by the analytical solution to a given real data set (Bayuk et al., 2007; Alkhimenkov and Bayuk, 2017).

\section{Frequency-dependent fluid bulk modulus}

Gurevich et al. (2010) show that the stiffness of the crack in relaxed and unrelaxed states can be described by the frequencydependent fluid bulk modulus $K_{f}^{*}(\omega)$. Our numerical results confirmed that this is true if the crack having $P_{f}=0$ boundary condition at the edge is embedded into the grain material whereas the torus is replaced by the grain material. This occurs because the Gurevich et al. (2010) solution for the frequency-dependent fluid bulk modulus applies boundary conditions to the walls of the crack and does not take into account the stiffness of the pore space surrounding the crack. Our numerical simulation of the modified frame clearly shows that once we include a small volume $(0.3 \%)$ of the dry stiff pore connected to the crack, the frequency dependence of the effective properties changes completely (Figure 11). However, it is very difficult to fully solve that coupled elasticity-fluid flow problem analytically.

The slopes of the high-frequency asymptote of the attenuation curve in the numerical and analytical solutions are different $\left(\omega^{-1}\right.$ for the analytical solution versus $\omega^{-1 / 2}$ for the numerical solution). In both numerical models, the big pore and the small pore models, the frequency range over which attenuation is significant broadens compared to the analytical solution due to the different asymptotic behavior of the attenuation curve in the high-frequency regime. It is interesting that the $\omega^{-1 / 2}$ power law also describes the highfrequency asymptote of the attenuation curves due to flow between stiff pores and mesoscopic cracks (i.e., cracks much larger than pores but still smaller than the wavelength) (Galvin and Gurevich, 2009; Guo et al., 2017a).

\section{The $C_{13}$ component of the frame moduli}

Our numerical simulations show that the $C_{13}$ component of the modified frame (the big pore model) is frequency dependent. Furthermore, the crack connected to the torus cannot be accurately described by only two parameters in the compliance domain: $Z_{n}$ and $Z_{t}$. Equation 17 shows the difference in the compliance domain $\Delta S_{m n}=S_{1}^{\mathrm{VTI}}-S_{3}^{\mathrm{VTI}}$, which is used to calculate $Z_{n}$ and $Z_{t}$ in workflow B (Figure 6). It can be seen that $\Delta S_{33}=4.251 \times 10^{-12}$ and $\Delta S_{44}=\Delta S_{55}=7.680 \times 10^{-12}$ are the actual $Z_{n}$ and $Z_{t}$ parameters in the workflow B. But $\Delta S_{13}=0.486 \times 10^{-12}$ is still significant. Hence, if we ignore the nonnegligible off-diagonal elements, the corresponding $C_{13}$ in the stiffness domain diverges from the correct numerical $C_{13}$ by approximately $4.5 \mathrm{GPa}$. This affects the analytical model in two ways. First, the difference between the low- and high-frequency limits of the $C_{13}$ component of the modified frame is approximately $4.5 \mathrm{GPa}$ in the big pore model, so the modified frame $C_{13}$ component gradually increases with frequency during the transition from the low to the high frequency. Second, in the generalized Gassmann's equation (equation 16), the calculation of $K^{*}$ involves the $C_{13}$ component four times (because $C_{13}=C_{23}=C_{31}=C_{32}$ ), which leads to more than $15 \mathrm{GPa}$ overestimation of the correct $K^{*}$ (thus, $K^{*}$ is also frequency dependent). Therefore, if we neglect the frequency dependence of the $C_{13}$ component, the generalized bulk modulus of the frame will become significantly different from the actual value. It is clearly visible in Figure 7 in which the low-frequency limit of the analytical solution with workflow B does not match the numerical result because this analytical solution is saturated using Gassmann's equations. However, the low-frequency limit of the modified frame for the analytical solution with workflow B is in excellent agreement with the numerical result because the stiff pore is dry, and the $C_{13}$ component effects vanish (Figure 11). For the big pore model,

$$
\begin{aligned}
\Delta S_{m n}^{\text {bigpore }}= & S_{1}^{\mathrm{VTI}}-S_{3}^{\mathrm{VTI}=}=10^{-12} \\
& \cdot\left[\begin{array}{cccccc}
0.034 & -0.037 & 0.486 & 0 & 0 & 0 \\
-0.037 & 0.034 & 0.486 & 0 & 0 & 0 \\
0.486 & 0.486 & 4.251 & 0 & 0 & 0 \\
0 & 0 & 0 & 7.680 & 0 & 0 \\
0 & 0 & 0 & 0 & 7.680 & 0 \\
0 & 0 & 0 & 0 & 0 & 0.018
\end{array}\right] .
\end{aligned}
$$


For the small pore model,

$\begin{aligned} \Delta S_{m n}^{\text {smallpore }}= & S_{1}^{\mathrm{VTI}}-S_{3}^{\mathrm{VTI}}=10^{-12} \\ & \cdot\left[\begin{array}{cccccc}0.004 & -0.0001 & 0.009 & 0 & 0 & 0 \\ -0.0001 & 0.004 & 0.009 & 0 & 0 & 0 \\ 0.009 & 0.009 & 2.244 & 0 & 0 & 0 \\ 0 & 0 & 0 & 3.109 & 0 & 0 \\ 0 & 0 & 0 & 0 & 3.109 & 0 \\ 0 & 0 & 0 & 0 & 0 & 0.010\end{array}\right] .\end{aligned}$

For the small pore model, the $C_{13}$ component is almost the same as for the $P_{f}=0$ model and does not change significantly over the frequency range. Equation 18 shows the difference in the compliance domain $\Delta S_{m n}=S_{1}^{\mathrm{VTI}}-S_{3}^{\mathrm{VTI}}$, which is used to calculate $Z_{n}$ and $Z_{t}$ in workflow B (see Figure 6). It can be seen that $\Delta S_{33}=$ $2.244 \times 10^{-12}$ and $\Delta S_{44}=\Delta S_{55}=3.109 \times 10^{-12}$ are the actual $Z_{n}$ and $Z_{t}$ parameters in workflow B. $\Delta S_{13}=0.009 \times 10^{-12}$ is negligible.

In summary, the crack compliance in the $P_{f}=0$ model and in the small pore model can be accurately described by only the two parameters: $Z_{n}$ and $Z_{t}$. But, in the big pore model, the crack compliance cannot be described by only $Z_{n}$ and $Z_{t}$, the $C_{13}$ component is also important.

\section{Fluid pressure field as a function of frequency}

One of the key parameters in the squirt flow mechanism is the ratio between the noncompliant and compliant pore volumes. In the big pore model, the ratio of noncompliant (torus) to compliant (crack) porosity is $0.045 /\left(4.9 \times 10^{-4}\right) \approx 92$. Therefore, only a small increase in the fluid pressure is expected in the big torus at low frequencies. In the big torus, the fluid pressure is $\approx 1 \times 10^{5} \mathrm{~Pa}$ in the low-frequency limit $\left(10^{0} \mathrm{~Hz}\right)$; the fluid pressure is $\approx 0.435 \times$ $10^{5} \mathrm{~Pa}$ in the high-frequency limit $\left(10^{6} \mathrm{~Hz}\right)$. Thus, the increase in fluid pressure is of approximately 2.3 times. The applied boundary conditions are for displacement $\left(10^{-6} \mathrm{~m}\right)$, and the fluid pressure values are given for the P-wave modulus test in $z$-direction (i.e., for $C_{33}$ component).

In the small pore model, the ratio of noncompliant to compliant porosity is $0.0034 /\left(4.9 \times 10^{-4}\right) \approx 7$. Thus, a significant increase in fluid pressure in the torus is expected in the low-frequency regime, compared to the high-frequency limit because the difference between noncompliant and compliant porosity is small (less than one order of magnitude). In the small torus, the fluid pressure is $\approx 3.3 \times 10^{5} \mathrm{~Pa}$ in the low-frequency limit $\left(10^{0} \mathrm{~Hz}\right)$; the fluid pressure is $\approx 0.58 \times 10^{5} \mathrm{~Pa}$ in the high-frequency limit $\left(10^{6} \mathrm{~Hz}\right)$. Thus, the increase in the fluid pressure is of approximately 5.7 times.

\section{CONCLUSION}

We have numerically calculated the frequency dependence of the effective stiffness properties of a fluid-saturated porous medium caused by squirt flow. Our 3D numerical models consist of a pore space embedded into a solid grain material. The pore space is represented by a flat cylinder, representing a crack, whose edge is connected with a torus, representing a stiff isometric pore. Grains were described as a linear isotropic elastic material, whereas the fluid phase filling the pore space was described by the quasistatic linearized compressible Navier-Stokes momentum equation.

We compared the numerical results to a published analytical solution for squirt flow. The numerical and analytical solutions agree only for the simplest model: The edge of the crack is subjected to zero fluid pressure boundary condition, whereas the stiff pore is absent. For this model, the low- and high-frequency limits of the dispersion and attenuation curves, intermediate-frequency behavior, and all asymptotes are in very good agreement between the numerical and analytical solutions. However, the considered model is not realistic.

For the model with a stiff pore modeled as a torus, there are significant differences between the numerical and analytical solutions: (1) the maximum attenuation predicted by the analytical model is significantly under- or overestimated, depending on the frame moduli calculation in the analytical model, (2) the transition from the low to high frequencies is much sharper compared with the numerical results, and (3) the slopes of the high-frequency asymptote of the attenuation curve in the numerical and analytical solutions are different $\left(\omega^{-1}\right.$ for the analytical solution versus $\omega^{-1 / 2}$ for the numerical solution).

Our analysis suggests that there are two main sources of discrepancies between the numerical results and the analytical solution. First, the calculation of the frame stiffness moduli in the analytical solution does not take into account the fact that pores and cracks are connected because it assumes a porous background and in the next step embeds cracks into this homogenized medium. The numerical results show that this two-step homogenization approach corresponds to the double-porosity media where the crack and pores are disconnected, which contradicts the concept of fluid flow in the analytical solution. Second, the frequency dependence of the attenuation and dispersion at intermediate frequencies in the analytical solution is based on several strong assumptions: The analytical model assumes that the frequency dependence is controlled only by the fluid flow in the crack, whereas the numerical results show that the frequency dependence is also affected by surrounding stiff pores connected to the crack. Further research should involve the modification of the analytical solution to include more adequate frame moduli calculation of the double-porosity model, more adequate modeling of the flow between compliant and stiff pores, and a more realistic geometry of the pore space.

\section{ACKNOWLEDGMENTS}

This research is funded by the Swiss National Science Foundation, project number 172691. Y. Alkhimenkov thanks H. Steeb for fruitful discussions regarding the numerical simulations and Y. Podladchikov for fruitful discussions regarding the Bessel functions. We thank Y. Guéguen, E. Saenger, and an anonymous reviewer for their constructive comments that helped us to improve the manuscript.

\section{DATA AND MATERIALS AVAILABILITY}

This is a theoretical study. Data associated with this research can be obtained by contacting the corresponding author. 


\section{APPENDIX A BOUNDARY CONDITIONS}

Let us consider a cuboid with volume $V=(0, L x) \times(0, L y) \times$ $(0, L z)$ and $\Gamma$ its boundary $\Gamma=\Gamma^{x z 0} \cup \Gamma^{x z L} \cup \Gamma^{y z 0} \cup \Gamma^{y z L} \cup$ $\Gamma^{x y 0} \cup \Gamma^{x y L}$, where, for example, $\Gamma^{x z 0}$ represents a $x z$-plane with zero coordinate and, e.g., $\Gamma^{x z L}$ represents a $x z$-plane with $L y$ coordinate. There are six planes in total. Because we are dealing with a VTI medium, only five independent components of the stiffness tensor are needed. Three kinds of tests were applied: a normal compression relaxation test, a simple shear test, and one mixed test for the $C_{13}$ component. The general approach is the following: We apply displacement boundary conditions for a certain frequency. Then, we calculate the volume average stress and strain fields over the whole model domain using the following equations for a given frequency $\omega$ :

$$
\left\langle\epsilon_{i j}\right\rangle=\frac{1}{V} \int_{V} \epsilon_{i j}(\mathrm{x}) \mathrm{dV},
$$

where $V$ is a model domain and

$$
\left\langle\sigma_{i j}\right\rangle=\frac{1}{V} \int_{V} \sigma_{i j}(\mathrm{x}) \mathrm{dV}
$$

Volume-averaged stress $\left\langle\sigma_{i j}\right\rangle$ and strain $\left\langle\epsilon_{i j}\right\rangle$ fields are related via the Hooke's law

$$
\left\langle\sigma_{i j}\right\rangle=C_{i j k l}:\left\langle\epsilon_{k l}\right\rangle
$$

which is the $C_{i j k l}=C_{m n}$ component of the stiffness matrix. Thus, we need several tests to obtain a relation between different $\left\langle\sigma_{i j}\right\rangle$ and $\left\langle\epsilon_{k l}\right\rangle$.

\section{Normal compression relaxation test}

Normal compression relaxation test is needed to calculate $M=\lambda+2 \mu$ component of the stiffness tensor.

For the $C_{33}$ component $\left(\left\langle\sigma_{3}\right\rangle=C_{33} \cdot\left\langle\epsilon_{3}\right\rangle\right)$ :

$\Gamma^{x y L}$ is set to $u_{z z}=\Delta u ; u_{x x}, u_{y y}$ are free

$\Gamma^{x y 0}$ is set to $u_{z z}=0 ; u_{x x}, u_{y y}$ are free

$\Gamma^{x z 0}$ and $\Gamma^{x z L}$ are set to $u_{y y}=0 ; u_{z z}, u_{x x}$ are free

$\Gamma^{y z 0}$ and $\Gamma^{y z L}$ are set to $u_{x x}=0 ; u_{z z}, u_{y y}$ are free,

where $\Delta u=10^{-6}$. The same relaxation tests are used to calculate $C_{11}$ and $C_{22}$ components.

\section{Simple shear relaxation test}

A simple shear relaxation test is needed to calculate the shear modulus $\mu$ component of the stiffness tensor.
For the $C_{55}(x z)$ component $\left(\left\langle\sigma_{5}\right\rangle=C_{55} \cdot\left\langle\epsilon_{5}\right\rangle\right)$,

$\Gamma^{x y L}$ is set to $u_{x x}=\Delta u ; u_{z z}, u_{y y}$ are free,

$\Gamma^{x y 0}$ is set to $u_{z z}=0 ; u_{x x}=0, u_{y y}=0$,

$\Gamma^{x z 0}$ and $\Gamma^{x z L}$ are set to $u_{y y}=0 ; u_{z z}=0, u_{x x}$ is free,

$\Gamma^{y z 0}$ and $\Gamma^{y z L}$ are set to $u_{y y}=0 ; u_{z z}=0, u_{x x}$ is free.

The same type of relaxation tests are used to calculate the $C_{44}$ and $C_{66}$ components.

\section{The $C_{13}$ mixed test}

The mixed test for $C_{13}$ component can be easily derived from the anisotropic stress-strain relation (Hooke's law) (similar to the 2D approach by Carcione et al., 2011).

$$
\begin{aligned}
& \Gamma^{x y L} \text { is set to } u_{z z}=\Delta u ; u_{x x}, u_{y y} \text { are free, } \\
& \Gamma^{x z L} \text { is set to } u_{y y}=\Delta u ; u_{z z}, u_{x x} \text { are free, } \\
& \Gamma^{x y 0} \text { is set to } u_{z z}=0 ; u_{x x}, u_{y y} \text { are free, } \\
& \Gamma^{x z 0} \text { is set to } u_{y y}=0 ; u_{x x}, u_{z z} \text { are free, } \\
& \Gamma^{y z 0} \text { and } \Gamma^{y z L} \text { are set to } u_{x x}=0 ; u_{z z}, u_{y y} \text { are free, }
\end{aligned}
$$

Then, using the following equations:

$$
C_{13}=\frac{\left\langle\sigma_{2}\right\rangle \cdot C_{33}-\left\langle\sigma_{3}\right\rangle \cdot C_{22}}{\left\langle\sigma_{3}\right\rangle-\left\langle\sigma_{2}\right\rangle},
$$

or

$$
C_{13}=\frac{\left\langle\sigma_{2}\right\rangle}{\left\langle\epsilon_{2}\right\rangle}-C_{22},
$$

the $C_{13}$ component is calculated $\left(C_{33}\right.$ and $C_{22}=C_{11}$ are taken from the direct tests). Equations A-7 and A- 8 are found from the Hooke's law considering nonzero strains in $y$ - and $z$-directions and, then, solving a system of two equations analytically.

\section{Direct test approach}

The direct test approach for calculating effective stiffness moduli can be used when the model's symmetry is known, which is the case in our modeling. According to the stress-strain relation (equation A-3), $C_{m n}$ components are calculated via

$$
C_{m n}=\frac{\left\langle\sigma_{m}\right\rangle}{\left\langle\epsilon_{n}\right\rangle} .
$$

We are dealing with a VTI medium, so only five independent components of the stiffness tensor are needed. Thus, we need two normal compression relaxation tests for $C_{11}$ and $C_{33}$ components (because $C_{11}=C_{22}$ ), two simple shear relaxation tests for $C_{55}$ and $C_{66}$ components (because $C_{44}=C_{55}$ ), and one mixed direct test for the $C_{13}$ component. 


\section{APPENDIX B}

\section{ROTATION OF A FOURTH-RANK TENSOR: HTI VERSUS VTI}

The stiffness matrix of a VTI medium is (in Voigt notation) (Tsvankin, 2012)

$C_{m n}^{\mathrm{VTI}}=\left[\begin{array}{cccccc}C_{11}^{\mathrm{VTI}} & C_{11}^{\mathrm{VTI}}-2 C_{66}^{\mathrm{VTI}} & C_{13}^{\mathrm{VTI}} & 0 & 0 & 0 \\ C_{11}^{\mathrm{VTI}}-2 C_{66}^{\mathrm{VTI}} & C_{11}^{\mathrm{VTI}} & C_{13}^{\mathrm{VTI}} & 0 & 0 & 0 \\ C_{13}^{\mathrm{VTI}} & C_{13}^{\mathrm{VTI}} & C_{33}^{\mathrm{VTI}} & 0 & 0 & 0 \\ 0 & 0 & 0 & C_{44}^{\mathrm{VTI}} & 0 & 0 \\ 0 & 0 & 0 & 0 & C_{44}^{\mathrm{VTI}} & 0 \\ 0 & 0 & 0 & 0 & 0 & C_{66}^{\mathrm{VTI}}\end{array}\right]$,

where the superscript $(\cdot)^{\text {VTI }}$ means that the component belongs to VTI media. In this study, all numerical simulations are performed in VTI media. The analytical solution of Collet and Gurevich (2016) is developed for horizontal transverse isotropy media (HTI). The stiffness matrix of HTI media with the symmetry axis along $x$-direction is

$C_{m n}^{\mathrm{HTI}}=\left[\begin{array}{cccccc}C_{11}^{\mathrm{HTI}} & C_{13}^{\mathrm{HTI}} & C_{13}^{\mathrm{HTI}} & 0 & 0 & 0 \\ C_{13}^{\mathrm{HTI}} & C_{33}^{\mathrm{HTI}} & C_{33}^{\mathrm{HTI}}-2 C_{44}^{\mathrm{HTI}} & 0 & 0 & 0 \\ C_{13}^{\mathrm{HTI}} & C_{33}^{\mathrm{HTI}}-2 C_{44}^{\mathrm{HTI}} & C_{33}^{\mathrm{HTI}} & 0 & 0 & 0 \\ 0 & 0 & 0 & C_{44}^{\mathrm{HTI}} & 0 & 0 \\ 0 & 0 & 0 & 0 & C_{66}^{\mathrm{HTI}} & 0 \\ 0 & 0 & 0 & 0 & 0 & C_{66}^{\mathrm{HTI}}\end{array}\right]$

where the superscript $(\cdot)^{\mathrm{HTI}}$ means that the component belongs to HTI media. Generally speaking, the fourth rank stiffness tensor in Euclidean space can be rotated using Euler angles. Fortunately, VTI and HTI symmetry classes belong to a transversely isotropic (TI) medium, which has a single axis of rotational symmetry (Fedorov, 1968). Therefore, the transformation from HTI media to VTI, and vice versa, doesn't require any sophisticated operations. Thus, the transformation from the VTI stiffness matrix to the HTI stiffness matrix, and vice versa, can be done by interchanging indices 1 and 3 and slightly modifying the structure of the stiffness matrix. The recipe is as follows: (1) Using the analytical solution of Collet and Gurevich (2016), calculate the resulting stiffness matrix that is of an HTI symmetry (equation B-2) and (2) construct the VTI stiffness matrix using the components of the HTI stiffness matrix from step (1) by the following rule:

$C_{m n}^{\mathrm{VTI}}=\left[\begin{array}{c}C_{33}^{\mathrm{HTI}} \\ C_{33}^{\mathrm{HTI}}-2 C_{44}^{\mathrm{HTI}} \\ C_{13}^{\mathrm{HTI}} \\ 0 \\ 0 \\ 0\end{array}\right.$

$C_{33}^{\mathrm{HTI}}-2 C_{44}^{\mathrm{HTI}}$
$C_{33}^{\mathrm{HTI}}$
$C_{13}^{\mathrm{HTI}}$
0
0
0

$\begin{array}{cc}C_{13}^{\mathrm{HTI}} & 0 \\ C_{13}^{\mathrm{HTI}} & 0 \\ C_{11}^{\mathrm{HTI}} & 0 \\ 0 & C_{66}^{\mathrm{HTI}} \\ 0 & 0 \\ 0 & 0\end{array}$

$\left.\begin{array}{cc}0 & 0 \\ 0 & 0 \\ 0 & 0 \\ 0 & 0 \\ C_{66}^{\mathrm{HTI}} & 0 \\ 0 & C_{44}^{\mathrm{HTI}}\end{array}\right]$

The resulting stiffness matrix (equation B-3) corresponds to VTI media, but all components are those from the HTI stiffness matrix (equation B-2).

\section{REFERENCES}

Alkhimenkov, Y., and I. Bayuk, 2017, Unified effective medium theory for cracked porous rocks - Theory and case study: 79th Annual International Conference and Exhibition, EAGE, Extended Abstracts, doi: 10 .3997/2214-4609.201701106.

Bakulin, A., V. Grechka, and I. Tsvankin, 2000a, Estimation of fracture parameters from reflection seismic data - Part 1: HTI model due to a single fracture set: Geophysics, 65, 1788-1802, doi: 10.1190/1.1444863.

Bakulin, A., V. Grechka, and I. Tsvankin, 2000b, Estimation of fracture parameters from reflection seismic data - Part 2: Fractured models with orthorhombic symmetry: Geophysics, 65, 1803-1817, doi: 10.1190/1 .1444864 .

Bayuk, I. O., M. Ammerman, and E. M. Chesnokov, 2007, Elastic moduli of anisotropic clay: Geophysics, 72, no. 5, D107-D117, doi: 10.1190/1 .2757624

Berryman, J. G., 2007, Seismic waves in rocks with fluids and fractures: Geophysical Journal International, 171, 954-974, doi: 10.1111/j.1365246X.2007.03563.x.

Biot, M. A., 1956, Theory of propagation of elastic waves in a fluidsaturated porous solid - 1: Low-frequency range: The Journal of the Acoustical Society of America, 28, 168-178, doi: 10.1121/1.1908239.

Biot, M. A., 1962, Generalized theory of acoustic propagation in porous dissipative media: The Journal of the Acoustical Society of America, 34, 1254-1264, doi: 10.1121/1.1918315.

Brajanovski, M., B. Gurevich, and M. Schoenberg, 2005, A model for P-wave attenuation and dispersion in a porous medium permeated by aligned fractures: Geophysical Journal International, 163, 372-384, doi: $10.1111 / j .1365-246 X .2005 .02722 . x$.

Carcione, J., J. Santos, and S. Picotti, 2011, Anisotropic poroelasticity and wave-induced fluid flow: Harmonic finite-element simulations: Geophysical Journal International, 186, 1245-1254, doi: 10.1111/j.1365246X.2011.05101.x.

Chapman, M., 2003, Frequency-dependent anisotropy due to meso-scale fractures in the presence of equant porosity: Geophysical Prospecting, 51, 369-379, doi: 10.1046/j.1365-2478.2003.00384.x.

Chapman, M., S. V. Zatsepin, and S. Crampin, 2002, Derivation of a microstructural poroelastic model: Geophysical Journal International, 151, 427-451, doi: 10.1046/j.1365-246X.2002.01769.x.

Chapman, S., J. V. Borgomano, H. Yin, J. Fortin, and B. Quintal, 2019, Forced oscillation measurements of seismic wave attenuation and stiffness moduli dispersion in glycerine-saturated Berea sandstone: Geophysical Prospecting, 67, 956-968, doi: 10.1111/1365-2478.12710.

Collet, O., and B. Gurevich, 2016, Frequency dependence of anisotropy in fluid saturated rocks - Part 1: Aligned cracks case: Geophysical Prospecting, 64, 1067-1084, doi: 10.1111/1365-2478.12384.

Das, V., T. Mukerji, and G. Mavko, 2019, Numerical simulation of coupled fluid-solid interaction at the pore scale: A digital rock-physics technology: Geophysics, 84, no. 4, WA71-WA81, doi: 10.1190/geo2018-0488.1.

Dvorkin, J., G. Mavko, and A. Nur, 1995, Squirt flow in fully saturated rocks: Geophysics, 60, 97-107, doi: 10.1190/1.1443767.

Fedorov, F. I., 1968, Theory of elastic waves in crystals: Springer.

Galvin, R., and B. Gurevich, 2009, Effective properties of a poroelastic medium containing a distribution of aligned cracks: Journal of Geophysical Research: Solid Earth, 114, B07305, doi: 10.1029/2008JB006032.

Gassmann, F., 1951, Über die elastizität poröser medien: Vierteljahrsschrift der Naturforschenden Gesellschaft in Zürich, 96, 1-23.

Grab, M., B. Quintal, E. Caspari, H. Maurer, and S. Greenhalgh, 2017, Numerical modeling of fluid effects on seismic properties of fractured magmatic geothermal reservoirs: Solid Earth, 8, 255-279, doi: 10 $.5194 / \mathrm{se}-8-255-2017$.

Guo, J., J. Germán Rubino, N. D. Barbosa, S. Glubokovskikh, and B. Gurevich, 2017a, Seismic dispersion and attenuation in saturated porous rocks with aligned fractures of finite thickness: Theory and numerical simulations - Part 1: P-wave perpendicular to the fracture plane: Geophysics, 83, no. 1, WA49-WA62, doi: 10.1190/geo2017-0065.1.

Guo, J., J. G. Rubino, S. Glubokovskikh, and B. Gurevich, 2017b, Effects of fracture intersections on seismic dispersion: Theoretical predictions versus numerical simulations: Geophysical Prospecting, 65, 1264-1276, doi: $10.1111 / 1365-2478.12474$

Gurevich, B., M. Brajanovski, R. J. Galvin, T. M. Müller, and J. TomsStewart, 2009a, P-wave dispersion and attenuation in fractured and porous reservoirs - Poroelasticity approach: Geophysical Prospecting, 57, 225-237, doi: 10.1111/j.1365-2478.2009.00785.x.

Gurevich, B., D. Makarynska, O. B. de Paula, and M. Pervukhina, 2010, A simple model for squirt-flow dispersion and attenuation in fluidsaturated granular rocks: Geophysics, 75, no. 6, N109-N120, doi: 10 $.1190 / 1.3509782$.

Gurevich, B., D. Makarynska, and M. Pervukhina, 2009b, Ultrasonic moduli for fluid-saturated rocks: Mavko-Jizba relations rederived and generalized: Geophysics, 74, no. 4, N25-N30, doi: 10.1190/1.3123802. 
Jakobsen, M., and M. Chapman, 2009, Unified theory of global flow and squirt flow in cracked porous media: Geophysics, 74, no. 2, WA65-WA76, doi: $10.1190 / 1.3078404$

Jones, T. D., 1986, Pore fluids and frequency-dependent wave propagation in rocks: Geophysics, 51, 1939-1953, doi: 10.1190/1.1442050.

Kachanov, M., 1993, Elastic solids with many cracks and related problems: Advances in Applied Mechanics, 30, 259-445, doi: 10.1016/S0065-2156 (08)70176-5.

Kachanov, M., and I. Sevostianov, 2018, Micromechanics of materials, with applications: Springer.

Landau, L., and E. Lifshitz, 1959, Course of theoretical physics: Fluid mechanics: Pergamon Press 6.

Masson, Y. J., and S. R. Pride, 2007, Poroelastic finite difference modeling of seismic attenuation and dispersion due to mesoscopic-scale heterogeneity: Journal of Geophysical Research: Solid Earth, 112, B3204, doi: $10.1029 / 2006 J B 004592$.

Masson, Y. J., S. R. Pride, and K. Nihei, 2006, Finite difference modeling of Biot's poroelastic equations at seismic frequencies: Journal of Geophysical Research: Solid Earth, 111, B10305, doi: 10.1029/2006JB004366.

Mavko, G., and D. Jizba, 1991, Estimating grain-scale fluid effects on velocity dispersion in rocks: Geophysics, 56, 1940-1949, doi: 10.1190/ 1.1443005 .

Mavko, G., T. Mukerji, and J. Dvorkin, 2009, The rock physics handbook: Tools for seismic analysis of porous media: Cambridge University Press.

Mavko, G., and A. Nur, 1975, Melt squirt in the asthenosphere: Journal of Geophysical Research, 80, 1444-1448, doi: 10.1029/JB080i011p01444.

Mayr, S. I., and H. Burkhardt, 2006, Ultrasonic properties of sedimentary rocks: Effect of pressure, saturation, frequency and microcracks: Geophysical Journal International, 164, 246-258, doi: 10.1111/j.1365246X.2005.02826.x.

Mikhaltsevitch, V., M. Lebedev, and B. Gurevich, 2015, A laboratory study of attenuation and dispersion effects in glycerol-saturated Berea sandstone at seismic frequencies: 85th Annual International Meeting, SEG, Expanded Abstracts, 3085-3089, doi: 10.1190/segam2015-5898429.1.

Mukerji, T., and G. Mavko, 1994, Pore fluid effects on seismic velocity in anisotropic rocks: Geophysics, 59, 233-244, doi: 10.1190/1.1443585.

Müller, T. M., B. Gurevich, and M. Lebedev, 2010, Seismic wave attenuation and dispersion resulting from wave-induced flow in porous rocks - A review: Geophysics, 75, no. 5, 75A147-75A164, doi: 10.1190/1.3463417.

Müller, T. M., J. Toms-Stewart, and F. Wenzlau, 2008, Velocity-saturation relation for partially saturated rocks with fractal pore fluid distribution Geophysical Research Letters, 35, L09306, doi: 10.1029/2007GL033074.

Murphy, W. F., K. W. Winkler, and R. L. Kleinberg, 1986, Acoustic relaxation in sedimentary rocks: Dependence on grain contacts and fluid saturation: Geophysics, 51, 757-766, doi: 10.1190/1.1442128.

O'Connell, R. J., and B. Budiansky, 1977, Viscoelastic properties of fluidsaturated cracked solids: Journal of Geophysical Research, 82, 57195735, doi: 10.1029/JB082i036p05719.

O'Connell, R. J., and B. Budiansky, 1978, Measures of dissipation in viscoelastic media: Geophysical Research Letters, 5, 5-8, doi: 10.1029/ GL005i001p00005.

Palmer, I., and M. Traviolia, 1980, Attenuation by squirt flow in undersaturated gas sands: Geophysics, 45, 1780-1792, doi: 10.1190/1.1441065.

Pervukhina, M., B. Gurevich, D. N. Dewhurst, and A. F. Siggins, 2010 Applicability of velocity-stress relationships based on the dual porosity concept to isotropic porous rocks: Geophysical Journal International, 181, 1473-1479, doi: 10.1111/j.1365-246X.2010.04535.x.

Pimienta, L., J. Fortin, and Y. Gueguen, 2015a, Bulk modulus dispersion and attenuation in sandstones: Geophysics, 80, no. 2, D111-D127, doi: 10 $.1190 /$ geo2014-0335.1.

Pimienta, L., J. Fortin, and Y. Gueguen, 2015b, Experimental study of Young's modulus dispersion and attenuation in fully saturated sandstones: Geophysics, 80, no. 5, L57-L72, doi: 10.1190/geo2014-0532.1.
Pride, S. R., and J. G. Berryman, 2003a, Linear dynamics of double-porosity dual-permeability materials - 1: Governing equations and acoustic attenuation: Physical Review E, 68, 036603, doi: 10.1103/PhysRevE 68.036603.

Pride, S. R., and J. G. Berryman, 2003b, Linear dynamics of double-porosity dual-permeability materials -2 : Fluid transport equations: Physical Review E, 68, 036604, doi: 10.1103/PhysRevE.68.036604.

Pride, S. R., J. G. Berryman, and J. M. Harris, 2004, Seismic attenuation due to wave-induced flow: Journal of Geophysical Research: Solid Earth, 109, B01201, doi: 10.1029/2003JB002639.

Quintal, B., E. Caspari, K. Holliger, and H. Steeb, 2019, Numerically quantifying energy loss caused by squirt flow: Geophysical Prospecting, 67, 2196-2212, doi: 10.1111/gpr.v67.8.

Quintal, B., J. G. Rubino, E. Caspari, and K. Holliger, 2016, A simple hydromechanical approach for simulating squirt-type flow: Geophysics, 81, no. 4, D335-D344, doi: 10.1190/geo2015-0383.1.

Rubino, J. G., T. M. Müller, L. Guarracino, M. Milani, and K. Holliger 2014, Seismoacoustic signatures of fracture connectivity: Journal of Geophysical Research: Solid Earth, 119, 2252-2271, doi: 10.1002/ 2013 JB010567.

Saenger, E. H., O. S. Krüger, and S. A. Shapiro, 2004, Effective elastic properties of randomly fractured soils: 3D numerical experiments: Geophysical Prospecting, 52, 183-195, doi: 10.1111/j.1365-2478.2004 .00407.x

Sayers, C., and M. Kachanov, 1995, Microcrack-induced elastic wave anisotropy of brittle rocks: Journal of Geophysical Research: Solid Earth, 100, 4149-4156, doi: 10.1029/94JB03134.

Schenk, O., and K. Gärtner, 2004, Solving unsymmetric sparse systems of linear equations with PARDISO: Future Generation Computer Systems, 20, 475-487, doi: 10.1016/j.future.2003.07.011.

Schoenberg, M., and K. Helbig, 1997, Orthorhombic media: Modeling elastic wave behavior in a vertically fractured earth: Geophysics, 62, 1954-1974, doi: 10.1190/1.1444297.

Schoenberg, M., and C. M. Sayers, 1995, Seismic anisotropy of fractured rock: Geophysics, 60, 204-211, doi: 10.1190/1.1443748.

Sevostianov, I., and M. Kachanov, 1999, Compliance tensors of ellipsoidal inclusions: International Journal of Fracture, 96, 3-7, doi: 10.1023/A 1018712913071

Subramaniyan, S., B. Quintal, C. Madonna, and E. H. Saenger, 2015, Laboratory-based seismic attenuation in Fontainebleau sandstone: Evidence of squirt flow: Journal of Geophysical Research: Solid Earth, 120, 7526-7535, doi: 10.1002/2015JB012290.

Tsvankin, I., 2012, Seismic signatures and analysis of reflection data in anisotropic media: SEG.

Walsh, J., 1965, The effect of cracks on the compressibility of rock: Journal of Geophysical Research, 70, 381-389, doi: 10.1029/ JZ070i002p00381.

White, J. E, 1975, Computed seismic speeds and attenuation in rocks with partial gas saturation: Geophysics, 40, 224-232, doi: 10.1190/1.1440520.

White, J. E., N. Mihailova, and F. Lyakhovitsky, 1975, Low-frequency seismic waves in fluid-saturated layered rocks: The Journal of the Acoustical Society of America, 57, S30-S30, doi: 10.1121/1.1995164.

$\mathrm{Xu}, \mathrm{S}, 1998$, Modelling the effect of fluid communication on velocities in anisotropic porous rocks: International Journal of Solids and Structures, 35, 4685-4707, doi: 10.1016/S0020-7683(98)00090-0.

Yan, H., B. Dupuy, A. Romdhane, and B. Arntsen, 2019, $\mathrm{CO}_{2}$ saturation estimates a Sleipner (North Sea) from seismic tomography and rock physics inversion: Geophysics Prospecting, 67, 1055-1071, doi: 10.1111/ 1365-2478.12693.

Zhang, Y., and M. N. Toksöz, 2012, Computation of dynamic seismic responses to viscous fluid of digitized three-dimensional Berea sandstones with a coupled finite-difference method: The Journal of the Acoustical Society of America, 132, 630-640, doi: 10.1121/1.4733545. 